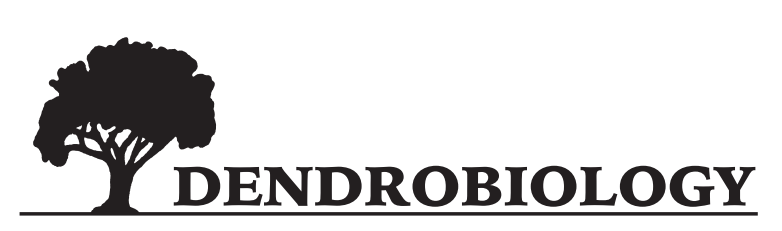

2018, vol. 80, 143-160

http://dx.doi.org/10.12657/denbio.080.014

\author{
Jolanta Behnke-Borowczyk, Hanna Kwaśna, Konrad Kokot, \\ Maciej Haluszczak, Piotr Łakomy
}

\title{
Abundance and diversity of fungi in oak wood
}

Received: 6 June 2018; Accepted: 24 January 2019

\begin{abstract}
Deadwood is an important functional and structural component of forest ecosystems since it regulates nutrient cycling, serves as a carbon (C) pool, increases the $\mathrm{C}$ content in the soil, and is a source of resources, including water, for a wide range of saproxylic and non-saproxylic organisms. The abundance and diversity of wood-inhabiting fungi in six fallen, horizontally lying logs of Quercus petraea, in the $1^{\text {st }}, 2^{\text {nd }}$ and $3^{\text {rd }}$ decay classes, in the Drawa National Park in Poland in 2015, were studied using the Illumina sequencing technique. The total number of OTUs obtained (264 307) included sequences of culturable fungi $(242369=91.70 \%)$ and non-culturable fungi $(7056=2.66 \%)$. The dead oak wood was colonized by 277 taxa. Culturable fungi of Zygomycota, Ascomycota and Basidiomycota comprised $75 \%$ of taxa detected and were represented by 14, 139 and 53 taxa respectively. Non-culturable organisms were represented by 70 taxa. Fungi were classified into 18 groups: (i) typical soft rot fungi, (ii) phytopathogens potentially hazardous to trees, (iii) epiphytes and endophytes or weak, opportunistic pathogens, (iv) ectomycorrhizal species, (v) ericoid mycorrhizal species, (vi) crust fungi, (vii) resupinate basidiomycetes, (viii) saprotrophs and soil fungi, (ix) opportunistic human pathogens causing superficial or systemic mycoses, (x) pathogens of warm- or cold-blooded animals, (xi) ascomycetous yeasts, (xii) basidiomycetous yeasts, (xiii) antagonists of fungi, (xiv) producers of metabolites effective against pathogens, (xv) hyperparasites, (xvi) lichen-forming species, (xvii) partners of other fungi, (xviii) species rare or uncommon in nature. It was shown that: (i) coarse, woody debris of oak is host to abundant and diverse mycobiota, (ii) abundance and diversity of fungi increase with the decay continuum, (iii) oak logs can be a habitat of phytopathogens potentially hazardous to forest health, (iv) different phytopathogens favour wood in different decay classes, (v) abundance of pathogens decreases, and of ericoid mycorrhizal (or soil) species increases with the decay continuum. Maintaining different types of deadwood increases fungal diversity, but also the risk of diseases.
\end{abstract}

Keywords: abundance, deadwood, diversity, fungi, ITS, Illumina, oak

Addresses: J. Behnke-Borowczyk, H. Kwaśna, K. Kokot, M. Hałuszczak, P. Łakomy, Poznan University of Life Sciences, Department of Forest Pathology, Wojska Polskiego 71c, 60-625 Poznań, Poland, e-mail: jbehnke@up.poznan.pl

\section{Introduction}

Deadwood is an important functional and structural component of forest ecosystems. It regulates nutrient cycling (Holub et al., 2001), serves as a carbon (C) pool (Harmon et al., 2004; Klein et al., 2013) and increases the $C$ content in the soil (Panayotov et al., 2016; Błońska et al., 2017). Additionally, it is an important source of water, especially during drought periods (Pichler et al., 2012). 
Deadwood is important resource for a wide range of saproxylic and non-saproxylic organisms, i.e. directly or indirectly dependent on dying or dead wood (Gutowski et al., 2004). Saproxylic species include many forest arthropods including beetles and other insects (Speight, 1989; Grove, 2002), and cavity-nesting birds (McComb \& Lindenmayer, 1999) and moluscs (Kappes et al., 2009). Non-saproxylic species include many small-bodied litter-dwelling invertebrates and vertebrates, including mammals such as dormice (Maser \& Trappe, 1984; Mac Nally et al., 2001; Fauteux et al., 2012), and amphibians (DeMaynadier \& Hunter, 1995). Deadwood is used as a habitat for many epixylic lichens and bryophytes (Andersson \& Hytteborn, 1991; Spribille et al., 2008). Tree seedlings on decayed logs benefit from recycled nutrients and microclimatic conditions (Szewczyk \& Szwagrzyk, 1996). Deadwood is also colonized by fungi, which are among the most diverse but least understood groups of saproxylic organisms (Boddy et al., 2008; Stokland et al., 2012).

The amount of deadwood that may naturally accumulate in forests varies and depends on the type of forest. In mixed mountain forest in central Europe it may rise to $216 \mathrm{~m}^{3}$ /ha (Hahn \& Christensen, 2005). In boreal forests in Fennoscandia, its volumes are $19-145 \mathrm{~m}^{3} / \mathrm{ha}$; in managed forests they are $2-10 \mathrm{~m}^{3}$ / ha (Siitonen, 2001). In Poland the average is $5.7 \mathrm{~m}^{3} /$ ha $\left(0-298 \mathrm{~m}^{3} / \mathrm{ha}\right)$ (Czerepko et al., 2008). The quantity of deadwood in Europe's forests has decreased significantly since the middle of the $19^{\text {th }}$ century due to intense forest exploitation and widespread burning of small wood and other debris (Forest Europe, 2011). Many European countries have launched initiatives to increase the amount of deadwood in forests. In Europe, in 1990-2005, this resulted in an increase in the amount of deadwood by about $4.3 \%$. It is widely agreed that $20-30 \mathrm{~m}^{3} / \mathrm{ha}$ is the required amount that can safeguard the complete spectrum of deadwood-dependent species (Siitonen, 2001; Angelstam et al., 2003; Humphrey et al., 2004; Vandekerkhove et al., 2009).

Wood-inhabiting fungi are key organisms for analysis and interpretation of the dynamics and conservation value of forests. However, there have been very few studies on the population and ecology of boreal wood-inhabiting fungi. Current knowledge of saproxylic fungi, their habitat preferences and succession during wood decomposition is based mainly on surveys of macroscopic fruit bodies (Allen et al., 2000; Ferris et al., 2000; Siitonen, 2001; Heilmann-Clausen \& Christensen 2004; Nordén et al., 2004; Vasiliauskas et al., 2004; Küffer \& Senn-Irlet, 2005; Lindner et al., 2006, 2011; Müller et al., 2007; Nordén et al., 2008; Fukasawa et al., 2009; Pouska et al., 2010; Fischer et al., 2012; Kebli et al., 2012; Ovaskainen et al., 2013). Only few studies included microfungi isolated on artificial culture media (Lumley et al., 2001; Fukasawa et al., 2009, 2011; Kwaśna et al., 2016a, b; 2017). Both approaches are selective; inconspicuous or non-fruiting species can be missed during surveys, and only a small portion of the microfungal community can be cultured in vitro. Recently, nucleic acid techniques have been applied to extract and identify fungal species colonizing deadwood in boreal forests (Allmér et al., 2006; Kulhánková et al., 2006; Ovaskainen et al., 2010; Rajala et al., 2010, 2011, 2012, 2015; Kebli et al., 2011; Lindner et al., 2011, van der Wal et al., 2017; Vaz et al., 2017). This approach is not restricted to any functional or taxonomic groups.

Although molecular techniques provide a more complete picture of fungal abundance and diversity and their relationship to substrate type and quality, the number of fungi involved in wood decay is still unknown. The percentage of fungal species dependent on deadwood is approximately $20-40 \%$ of the estimated 1.5 million fungal species worldwide (Siitonen, 2001; Penttilä et al., 2004). About 2000 species of wood-inhabiting fungi are known from the Nordic countries (Stokland \& Meyke, 2008).

Thus, the aims of this study were: (i) to characterize deadwood-inhabiting fungal communities according to specific wood attributes, i.e. type and quality (oak, logs, $60 \times 20 \times 20 \mathrm{~cm}$ samples, $1^{\text {st }}, 2^{\text {nd }}$ and $3^{\text {rd }}$ decay classes); (ii) to categorize fungi detected in/on oak deadwood in a single forest; (iii) to determine the ecological succession of fungi along the decay continuum; (iv) to determine possible interactions among fungi. The hypothesis that fungal abundance and diversity increase with the decay class of oak deadwood is tested.

\section{Material and methods}

\section{Sampling}

Samples of log deadwood (60 x $20 \times 20 \mathrm{~cm}$ ) were collected in one stand in Drawa National Park, Poland $\left(53^{\circ} 7^{\prime} 48^{\prime \prime} \mathrm{N}, 15^{\circ} 27^{\prime} 0^{\prime \prime} \mathrm{E}\right)$ in October 2015. The stand consisted of 85-year-old sessile oak (Quercus petraea (Matt. Liebl.), European beech (Fagus sylvatica L.), black alder (Alnus glutinosa Gaertn.) and isolated Scots pines (Pinus sylvestris L.). The shrub and herb layers included Agrostis stolonifera L., Calluna vulgaris (L.) Hull, Convallaria majalis L., Corynephorus canescens (L.) P. Beauv., Hepatica nobilis Mill., Poa pratensis L., Polypodium vulgare L., Polytrichum commune Hedw. and Vaccinium myrtillus L. Six samples of sessile oak deadwood were taken from six fallen, horizontally lying logs located $20-100 \mathrm{~m}$ apart. Two samples were in the $1^{\text {st }}$, two in the $2^{\text {nd }}$, and two in the $3^{\text {rd }}$ decay classes (Hunter, 1990). They were representative of logs decaying in the stand. Wood in the $1^{\text {st }}$ decay class (1-3 
years after felling) was sound, with intact structure and original colour, and the bark was intact. In the $2^{\text {nd }}$ decay class (5-20 years after felling), the wood was moderately soft, its colour had visibly changed, smaller or larger patches or rings were rotted, especially in the outer layers, and the bark had partially fallen off. In the $3^{\text {rd }}$ decay class (20-30 years after felling) the wood was soft, its colour had already changed, the core was often still solid, the outer layers disintegrated (knife test), and bark was absent.

In the laboratory, six sub-samples of sawdust were taken at $10-\mathrm{cm}$ intervals from each sample with a cordless SPARKY BUR2 15E drill (Hoppe et al., 2014). Sub-samples included bark (if present), heartwood and sapwood.

\section{DNA extraction, amplification and Illumina sequencing}

Six sub-samples of sawdust from each sample were pooled and ground in a SPEXTM SamplePrepTM Freezer/MillTM cryogenic mill. The total DNA was extracted with Plant Genomic DNA Purification Kit (Thermo Scientific). ITS 2 rDNA amplification was performed with fungus-specific primers: gITS7 (5' GTG ART CAT CGA RTC TTT G 3`; Ihrmark et al., 2012) and ITS4 (5`TCC TCC GCT TAT TGA TAT GC 3`; White et al., 1990). The PCR reaction mixture consisted of $12.5 \mu \mathrm{l}$ of $2 \mathrm{x}$ Mix PCR (A\&A Biotechnology), $0.2 \mu \mathrm{M}$ of each primer, $1.5 \mu \mathrm{l}$ of purified and diluted DNA and $10.6 \mu \mathrm{l}$ of water. DNA amplification was performed under the following conditions: denaturation at $94^{\circ} \mathrm{C}$ for $5 \mathrm{~min}$, followed by 35 cycles of denaturation at $94^{\circ} \mathrm{C}$ for 30 s, annealing at $56^{\circ} \mathrm{C}$ for $30 \mathrm{~s}$, elongation at $72^{\circ} \mathrm{C}$ for $30 \mathrm{~s}$, and a final elongation at $72^{\circ} \mathrm{C}$ for $7 \mathrm{~min}$. Visualization of $5 \mu \mathrm{l}$ amplicons was performed in 1.0\% agarose gel dyed with Midori Green Advance DNA (Genetics). The PCR products were purified with MinElute PCR Purification Kit (Qiagen). The amplicons were sequenced using the Illumina system in the Genomic Laboratory, DNA Research Center, Rubież 46, Poznań, Poland.

\section{Bioinformatics analysis}

A table of Operational Taxonomic Units (OTUs) was prepared by PIPITS, version 1.2.0 (Gweon et al., 2015). Read-pairs were joined with PEAR, version 0.9.6 (Zhang et al., 2014), filtered with a quality threshold of $\mathrm{q}=30$ by FASTX-toolkit, version 0.0 .13 (http://hannonlab.cshl.edu/fastx_toolkit/index. html), converted to Fasta format and merged into a single file. Prepared sequences were de-replicated and subregions of ITS were selected with the use of ITSx, version 1.0.11 (Bengtsson-Palme et al., 2013).
Unique sequences and those shorter than $100 \mathrm{bp}$ were removed. Remaining sequences were clustered with $97 \%$ sequence identity. The resulting representative sequences for each cluster were subjected to chimera detection and removal using the UNITE UCHIME reference dataset, version 6.0 (https://unite.ut.ee/ index.php). The input sequences were then mapped onto the representative sequences and taxonomy assigned using RDP Classifier, version 2.10.2 (Wang et al., 2007) against UNITE fungal ITS reference database, version 11.2 (Cole et al., 2014). This process resulted in the creation of a table of OTUs. Sequences were identified by comparison with reference sequences from the National Center for Biotechnology Information (NCBI) database.

Abundance of fungi was defined as the number of OTUs in a sample. Frequency of an individual taxon was defined as percentage (\%) of OTUs in the total number of OTUs. Diversity was defined as the number of species in a sample.

\section{Statistical analysis}

Diversity in microbial communities was compared with diversity indices calculated for each community, including their abundance and taxonomic composition (Magurran, 1988). Diversity in a community was indicated by Margalef's diversity index (DMg) and Shannon's diversity index $\left(\mathrm{H}^{\prime}\right)$. Evenness and dominance were indicated by Simpson's diversity index (D), Shannon's evenness index (E) and Berger-Parker's dominance index (d). The similarity between fungal communities was determined by calculating Sorensen's qualitative similarity index $(\mathrm{CN})$. Similarity and relationships among fungal communities are shown by a heat map and Venn diagram.

\section{Results}

In total, 264307 OTUs were obtained from the deadwood of six oak logs using the Illumina sequencing technique (Table 1). This number includes sequences of culturable fungi $(242369=91.70 \%)$, non-culturable fungi $(7056=2.66 \%)$ and organisms with no reference sequence in NCBI $(14880=$ $5.62 \%)$. The number of OTUs increased with the decay class.

Ninety percent of culturable taxa were linked to a genus or species. The frequency of Zygomycota was $0.4-3.9 \%$, Ascomycota was $81.3-89.2 \%$ and $\mathrm{Ba}-$ sidiomycota was $2.8-5.7 \%$. Wood was colonized by 277 taxa. Culturable Zygomycota, Ascomycota and Basidiomycota comprised $74.4 \%$ of all taxa detected and were represented by 14, 139 and 53 taxa, respectively. Non-culturable organisms were represented 
Table 1. Fungi in wood of oak

\begin{tabular}{|c|c|c|c|c|c|}
\hline \multirow{3}{*}{ No } & \multirow{3}{*}{ Taxa } & \multirow{3}{*}{ Order } & \multirow{2}{*}{\multicolumn{3}{|c|}{$\frac{\text { Frequency }(\%) \text { of OTUs }}{\text { Decay class }}$}} \\
\hline & & & & & \\
\hline & & & $1^{\mathrm{st}}$ & $2^{\text {nd }}$ & $3^{\text {rd }}$ \\
\hline 1 & Ambomucor seriatoinflatus X.Y. Liu \& R.Y. Zheng & Mucorales & 0.004 & 0.000 & 0.000 \\
\hline 2 & $\begin{array}{l}\text { Mortierella alpina Peyronel }+ \text { M. gamsii Milko }+ \text { M. macrocystopsis W. Gams } \\
\& \text { Carreiro }+ \text { M. parvispora Linnem. }+ \text { Mortierella sp. }\end{array}$ & Mortierellales & 0.035 & 0.046 & 0.036 \\
\hline 3 & $\begin{array}{l}\text { Mucor abundans Povah }+M \text {. fragilis Bainier }+ \text { M. genevensis Lendn. }+M \text {. } \\
\text { silvaticus Hagem }+ \text { M. racemosus Fresen. }\end{array}$ & Mucorales & 0.275 & 0.085 & 3.738 \\
\hline \multirow[t]{2}{*}{4} & $\begin{array}{l}\text { Umbelopsis isabellina (Oudem.) W. Gams + U. ramanniana (Möller) W. Gams } \\
+ \text { Umbelopsis sp. }\end{array}$ & Mucorales & 0.040 & 0.000 & 0.100 \\
\hline & Zygomycota & & 0.354 & 0.131 & 3.874 \\
\hline 1 & $\begin{array}{l}\text { Alternaria alternata (Fr.) Keissl. + A. infectoria E.G. Simmons + A. rosae E.G. } \\
\text { Simmons \& C.F. Hil }\end{array}$ & Pleosporales & 0.041 & 0.067 & 0.017 \\
\hline 2 & Archaeorhizomyces finlayi Rosling \& T.Y. James & Archaeorhizomycetales & 0.000 & 0.000 & 0.001 \\
\hline 3 & Arthoniomycetes & & 0.122 & 0.103 & 0.003 \\
\hline 4 & Ascocoryne sarcoides (Jacq.) J.W. Groves \& D.E. Wilson & Helotiales & 7.620 & 0.092 & 2.182 \\
\hline 5 & Ascomycota & & 0.008 & 0.000 & 0.023 \\
\hline 6 & $\begin{array}{l}\text { Aspergillus auratus Warcup + A. calidoustus Varga Houbraken \& Samson }+ \\
\text { A. cervinus Massee }+ \text { A. conicus Blochwitz }+ \text { A. fumigatus Fresen. }+ \text { A. halo- } \\
\text { philicus M. Chr.. Papav. \& C.R. Benj. }+ \text { A. kanagawaensis Nehira }+ \text { A. nutans } \\
\text { McLennan \& Ducker + A. parvulus G. Sm. }\end{array}$ & Eurotiales & 2.820 & 0.281 & 7.319 \\
\hline 7 & Aureobasidium pullulans (de Bary \& Löwenthal) G. Arnaud & Dothideales & 4.932 & 7.132 & 2.817 \\
\hline 8 & Babjeviella inositovora (Golubev \& Blagod.) Kurtzman \& M. Suzuki & Saccharomycetales & 0.000 & 0.000 & 0.125 \\
\hline 9 & Botryosphaeria stevensii Shoemaker & Botryosphaeriales & 18.140 & 0.126 & 0.016 \\
\hline 10 & Botrytis anthophila Bondartsev + B. cinerea Pers. & Helotiales & 0.027 & 13.411 & 0.003 \\
\hline 11 & Brachysporium nigrum (Link) S. Hughes & Trichosphaeriales & 0.023 & 0.053 & 0.013 \\
\hline 12 & $\begin{array}{l}\text { Cadophora finlandica (C.J.K. Wang \& H.E. Wilcox) T.C. Harr. \& McNew + } \\
\text { Cadophora sp. }\end{array}$ & Helotiales & 0.089 & 0.103 & 0.001 \\
\hline 13 & $\begin{array}{l}\text { Candida albicans (C.P. Robin) Berkhout }+ \text { C. zeylanoides (Castell.) Langeron } \\
\& \text { Guerra }+ \text { Candida sp. }\end{array}$ & Saccharomycetales & 0.030 & 0.000 & 0.552 \\
\hline 14 & Capronia pilosella (P. Karst.) E. Müll. Petrini P.J. Fisher Samuels \& Rossman & Chaetothyriales & 0.008 & 0.000 & 0.020 \\
\hline 15 & Cenangium acuum Cooke \& Peck & Helotiales & 0.000 & 0.010 & 0.011 \\
\hline 16 & Cenococcum geophilum Fr. & Mytilinidiales & 0.023 & 0.000 & 0.001 \\
\hline 17 & Chaetomiaceae & Sordariales & 0.023 & 0.038 & 0.015 \\
\hline 18 & $\begin{array}{l}\text { Chaetosphaeria decastyla (Cooke) Réblová \& W. Gams + C. vermicularioides } \\
\text { (Sacc. \& Roum.) W. Gams \& Hol.-Jech. + Chaetosphaeria sp. }\end{array}$ & Chaetosphaeriales & 0.004 & 0.000 & 0.390 \\
\hline 19 & Chaetothyriales & Chaetothyriales & 0.008 & 0.000 & 0.000 \\
\hline 20 & Chaetothyriomycetidae & & 0.000 & 0.002 & 0.000 \\
\hline 21 & $\begin{array}{l}\text { Cladophialophora chaetospira (Grove) Crous \& Arzanlou + Cladophialophora } \\
\text { sp. }\end{array}$ & Chaetothyriales & 0.004 & 0.002 & 0.024 \\
\hline 22 & $\begin{array}{l}\text { Cladosporium cladosporioides (Fresen.) G.A. de Vries + C. herbarum (Pers.) } \\
\text { Link + C. sphaerospermum Penz. + C. tenuissimum Cooke + Cladosporium sp. }\end{array}$ & Capnodiales & 6.096 & 23.300 & 0.022 \\
\hline 23 & $\begin{array}{l}\text { Colletotrichum capsici (Syd. \& P. Syd.) E.J. Butler \& Bisby + C. gloeosporioides } \\
\text { (Penz.) Penz. \& Sacc. }\end{array}$ & Glomerellales & 0.076 & 0.150 & 0.000 \\
\hline 24 & Cosmospora sp. & Hypocreales & 0.042 & 0.101 & 0.064 \\
\hline 25 & Debaryomyces hansenii (Zopf) Lodder \& Kreger-van Rij & Saccharomycetales & 0.000 & 0.000 & 0.002 \\
\hline 26 & $\begin{array}{l}\text { Dematioscypha catenata (Preuss) P.R. Johnst. + D. dematiicola (Berk. \& } \\
\text { Broome) Svrček }\end{array}$ & Helotiales & 0.042 & 0.000 & 0.037 \\
\hline 27 & Dermateaceae & Helotiales & 0.782 & 0.082 & 0.071 \\
\hline 28 & Didymella macrostoma (Mont.) Q. Chen \& L. Cai & Pleosporales & 0.000 & 0.073 & 0.000 \\
\hline 29 & Epicoccum nigrum Link & Pleosporales & 0.000 & 0.002 & 0.001 \\
\hline 30 & Erysiphe alphitoides (Griffon \& Maubl.) U. Braun \& S. Takam. & Erysiphales & 0.000 & 0.000 & 0.002 \\
\hline 31 & Eucasphaeria capensis Crous & Hypocreales & 0.000 & 0.002 & 0.000 \\
\hline 32 & Eurotiales & Eurotiales & 0.004 & 0.000 & 0.000 \\
\hline 33 & Exophiala angulospora Iwatsu Udagawa \& T. Takase & Chaetothyriales & 0.000 & 0.000 & 0.001 \\
\hline 34 & Fulgidea oligospora (Timdal) Bendiksby \& Timdal & Umbilicariales & 0.000 & 0.002 & 0.000 \\
\hline 35 & Fusarium lateritium Nees + F. oxysporum Schltdl. + F. roseum Link & Hypocreales & 0.544 & 0.021 & 0.019 \\
\hline 36 & Geomyces auratus Traaen + G. pannorum (Link) Sigler \& J.W. Carmich. & Incertae sedis & 1.340 & 0.000 & 16.655 \\
\hline 37 & Helotiales & Helotiales & 0.000 & 0.000 & 0.016 \\
\hline
\end{tabular}




\begin{tabular}{|c|c|c|c|c|c|}
\hline & \multirow{3}{*}{ Taxa } & \multirow{3}{*}{ Order } & \multirow{2}{*}{\multicolumn{3}{|c|}{$\begin{array}{c}\text { Frequency }(\%) \text { of OTUs } \\
\text { Decay class }\end{array}$}} \\
\hline & & & & & \\
\hline & & & $1^{\text {st }}$ & $2^{\text {nd }}$ & $3^{\text {rd }}$ \\
\hline 38 & Hyaloscypha albohyalina (P. Karst.) Boud. & Helotiales & 0.000 & 0.004 & 0.013 \\
\hline 39 & Hymenoscyphus sp. & Helotiales & 0.012 & 0.002 & 0.001 \\
\hline 40 & Hypoxylon rubiginosum (Pers.) Fr. & Xylariales & 0.000 & 0.044 & 0.012 \\
\hline 41 & Infundichalara microchona (W. Gams) Réblová \& W. Gams & Helotiales & 0.004 & 0.000 & 0.007 \\
\hline 42 & Lachnellula hyalina Dharne & Helotiales & 0.000 & 0.002 & 0.000 \\
\hline 43 & Lecythophora sp. & Coniochaetales & 23.560 & 0.258 & 0.095 \\
\hline 44 & Leotiomycetes & Leotiomycetes & 0.004 & 0.002 & 0.000 \\
\hline 45 & $\begin{array}{l}\text { Leptodontidium trabinellum (P. Karst.) Baral. Platas \& R. Galán + Leptodon- } \\
\text { tidium sp. }\end{array}$ & Helotiales & 0.447 & 0.058 & 1.311 \\
\hline 46 & Moristroma quercinum Nordén & Phaeomoniellales & 0.000 & 0.000 & 0.013 \\
\hline 47 & Natantiella ligneola (Berk. \& Broome) Réblová & Incertae sedis & 0.000 & 0.000 & 0.010 \\
\hline 48 & Nakazawaea holstii (Wick.) Y. Yamada. K. Maeda \& Mikata & Saccharomycetales & 0.004 & 0.000 & 0.000 \\
\hline 49 & Nectria cinnabarina (Tode) Fr. & Hypocreales & 0.019 & 0.044 & 0.002 \\
\hline 50 & Nemania serpens (Pers.) Gray & Xylariales & 0.000 & 0.000 & 0.004 \\
\hline 51 & Neocatenulostroma germanicum (Crous \& U. Braun) Quaedvl. \& Crous & Capnodiales & 0.096 & 0.199 & 0.010 \\
\hline 52 & $\begin{array}{l}\text { Oidiodendron chlamydosporicum Morrall }+ \text { O. periconioides Morrall }+ \text { O. rho- } \\
\text { dogenum Robak }+ \text { O. tenuissimum (Peck) S. Hughes }\end{array}$ & Incertae sedis & 0.015 & 0.136 & 3.285 \\
\hline 53 & Ophiostoma novo-ulmi Brasier & Ophiostomatales & 16.370 & 20.155 & 23.414 \\
\hline 54 & Orbilia coccinella Fr. + O. eucalypti (W. Phillips \& Harkn.) Sacc. & Orbiliales & 0.011 & 0.002 & 0.001 \\
\hline 55 & $\begin{array}{l}\text { Penicillium adametzii K.M. Zaleski + P. buchwaldii frisvad \& Samson + P. } \\
\text { canescens Sopp + } \\
\text { P. citreonigrum Dierckx + P. expansum Link + P. glabrum (Wehmer) Westling } \\
+ \text { P. lanosum Westling + P. longicatenatum Visagie Busby Houbraken \& K. } \\
\text { Jacobs + P. macrosclerotiorum L. Wang X.M. Zhang \& W.Y. Zhuang + P. } \\
\text { quercetorum Baghd. + P. rubens Biourge + P. saturniforme (L. Wang \& W.Y. } \\
\text { Zhuang) Houbraken \& Samson + P. solitum Westling + P. spinulosum Thom } \\
+ \text { P. thomii Maire + P. westlingii K.M. Zaleski }\end{array}$ & Eurotiales & 0.975 & 1.660 & 21.150 \\
\hline 56 & Pesotum piceae J.L. Crane \& Schokn. & Ophiostomatales & 0.000 & 0.029 & 0.001 \\
\hline 57 & Pestalotiopsis sp. & Xylariales & 0.023 & 0.006 & 0.021 \\
\hline 58 & Phaeosphaeria juncophila Leuchtm. & Pleosporales & 0.004 & 0.000 & 0.000 \\
\hline 59 & $\begin{array}{l}\text { Phialocephala fortinii C.J.K. Wang \& H.E. Wilcox + P. nodosa J.B. Tanney \& } \\
\text { B. Douglas }\end{array}$ & Helotiales & 0.840 & 0.008 & 0.024 \\
\hline 60 & Phialophora sp. & Chaetothyriales & 2.950 & 12.800 & 0.051 \\
\hline 61 & Phoma herbarum Westend & Pleosporales & 0.034 & 0.018 & 0.009 \\
\hline 62 & Phomopsis juglandina (Sacc.) Höhn. & Diaporthales & 0.076 & 0.034 & 0.003 \\
\hline 63 & Pleotrichocladium opacum (Corda) Hern.-Restr.. R.F. Castañeda \& Gené & Sordariales & 0.000 & 0.000 & 0.001 \\
\hline 64 & Pleurostoma richardsiae (Nannf.) Réblová \& Jaklitsch & Calosphaeriales & 0.000 & 0.000 & 0.002 \\
\hline 65 & Pseudogymnoascus pannorum (Link) Minnis \& D.L. Lindner + P. roseus Raillo & Incertae sedis & 0.545 & 0.002 & 0.158 \\
\hline 66 & Rhinocladiella atrovirens Nannf. & Chaetothyriales & 0.000 & 0.002 & 0.008 \\
\hline 67 & Rhizosphaera kalkhoffii Bubák & Dothideales & 0.243 & 0.486 & 0.950 \\
\hline 68 & $\begin{array}{l}\text { Saccharomyces cerevisiae Meyen ex E.C. Hansen }+ \text { S. pastorianus Reess ex } \\
\text { E.C. Hansen }\end{array}$ & Saccharomycetales & 0.035 & 0.000 & 0.316 \\
\hline 69 & $\begin{array}{l}\text { Sagenomella diversispora (J.F.H. Beyma) W. Gams + S. striatispora (Onions \& } \\
\text { G.L. Barron) W. Gams }\end{array}$ & Eurotiales & 0.000 & 0.000 & 0.002 \\
\hline 70 & Scheffersomyces shehatae (H.R. Buckley \& Uden) Urbina \& M. Blackw. & Saccharomycetales & 0.008 & 0.048 & 5.838 \\
\hline 71 & Scytalidium album L. Beyer \& Klingström & Incertae sedis & 0.000 & 0.006 & 0.004 \\
\hline 72 & Sporothrix sp. & Ophiostomatales & 0.008 & 0.002 & 0.092 \\
\hline 73 & Sugiyamaella paludigena (Golubev \& Blagod.) H. Urbina \& M. Blackw. & Saccharomycetales & 0.000 & 0.017 & 0.000 \\
\hline 74 & Sydowia polyspora (Bref. \& Tavel) E. Müll. & Dothideales & 0.004 & 0.017 & 0.001 \\
\hline 75 & $\begin{array}{l}\text { Talaromyces islandicus (Sopp) Samson N. Yilmaz Frisvad \& Seifert }+T \text {. } \\
\text { rugulosus (Thom) Samson N. Yilmaz Frisvad \& Seifert }+ \text { T. variabilis (Sopp) } \\
\text { Samson N. Yilmaz Frisvad \& Seifert + Talaromyces sp. }\end{array}$ & Eurotiales & 0.034 & 0.050 & 0.001 \\
\hline 76 & Taphrina purpurascens B.L. Rob. & Taphrinales & 0.011 & 0.000 & 0.000 \\
\hline 77 & Titaea maxilliformis Rostr. & Tubeufiales & 0.023 & 0.000 & 0.000 \\
\hline 78 & $\begin{array}{l}\text { Trichoderma asperellum Samuels Lieckf. \& Nirenberg }+ \text { T. minutisporum Bis- } \\
\text { sett }+ \text { T. paraviridescens Jaklitsch Samuels \& Voglmayr }+ \text { T. semiorbis (Berk.) } \\
\text { Jaklitsch \& Voglmayr }+ \text { T. sinuosum P. Chaverri \& Samuels }\end{array}$ & Hypocreales & 0.008 & 0.059 & 0.063 \\
\hline
\end{tabular}




\begin{tabular}{|c|c|c|c|c|c|}
\hline \multirow{3}{*}{ No } & \multirow{3}{*}{ Taxa } & \multirow{3}{*}{ Order } & \multirow{2}{*}{\multicolumn{3}{|c|}{$\begin{array}{c}\text { Frequency }(\%) \text { of OTUs } \\
\text { Decay class }\end{array}$}} \\
\hline & & & & & \\
\hline & & & $1^{\mathrm{st}}$ & $2^{\text {nd }}$ & $3^{\text {rd }}$ \\
\hline 79 & Xenoacremonium falcatum L. Lombard \& Crous & Hypocreales & 0.000 & 0.000 & 0.01 \\
\hline \multicolumn{3}{|c|}{ Ascomycota } & 89.203 & 81.303 & 87.306 \\
\hline 1 & Agaricomycotina & & 0.000 & 0.000 & 2.912 \\
\hline 2 & Amanita rubescens Pers. & Agaricales & 0.000 & 0.067 & 0.000 \\
\hline 3 & Armillaria cepistipes Velen.+ A. gallica Marxm. \& Romagn. & Agaricales & 0.863 & 2.285 & 0.478 \\
\hline 4 & Asterotremella sp. & Tremellales & 0.000 & 0.000 & 1.025 \\
\hline 5 & Athelia acrospora Jülich & Atheliales & 0.065 & 0.000 & 0.002 \\
\hline 6 & Baeospora sp. & Agaricales & 0.019 & 0.000 & 0.000 \\
\hline 7 & Basidiomycete & & 0.027 & 0.040 & 0.006 \\
\hline 8 & Bjerkandera adusta (Willd.) P. Karst. & Polyporales & 0.000 & 0.059 & 0.012 \\
\hline 9 & Chlorophyllum olivieri (Barla) Vellinga & Agaricales & 0.000 & 0.000 & 0.005 \\
\hline 10 & Cryptococcus tephrensis Vishniac + Cryptococcus sp. & Tremellales & 0.000 & 0.174 & 0.005 \\
\hline 11 & Cuniculitrema polymorpha R. Kirschner \& J.P. Samp. & Tremellales & 0.000 & 0.000 & 0.002 \\
\hline 12 & Dacrymycetes & & 0.000 & 0.038 & 0.000 \\
\hline 13 & Daedaleopsis confragosa (Bolton) J. Schröt. & Polyporales & 0.027 & 0.000 & 0.000 \\
\hline 14 & Dioszegia hungarica Zsolt & Tremellales & 0.015 & 0.000 & 0.000 \\
\hline 15 & Entocybe turbida (Fr.) T. J. Baroni V. Hofstetter \& Largent & Agaricales & 0.030 & 0.120 & 0.019 \\
\hline 16 & $\begin{array}{l}\text { Filobasidium wieringae (Á. Fonseca. Scorzetti \& Fell) Xin Zhan Liu. F.Y. Bai. } \\
\text { M. Groenew. \& Boekhout }\end{array}$ & Filobasidiales & 0.042 & 0.000 & 0.000 \\
\hline 17 & Ganoderma applanatum (Pers.) Pat. & Polyporales & 0.000 & 0.000 & 0.013 \\
\hline 18 & $\begin{array}{l}\text { Genolevuria armeniaca (Á. Fonseca \& J. Inácio) Xin Zhan Liu. F.Y. Bai. M. } \\
\text { Groenew. \& Boekhout }\end{array}$ & Tremellales & 0.021 & 0.000 & 0.000 \\
\hline 19 & $\begin{array}{l}\text { Hamamotoa lignophila (I. Dill. C. Ramírez \& A.E. González) Q.M. Wang. } \\
\text { F.Y. Bai. M. Groenew. \& Boekhout }\end{array}$ & Sporidiobolales & 0.011 & 0.000 & 0.001 \\
\hline 20 & Hebeloma pamphiliense Cittadini Lezzi \& Contu & Agaricales & 0.019 & 0.000 & 0.000 \\
\hline 21 & Hymenogaster pruinatus $\mathrm{R}$. Hesse & Agaricales & 0.000 & 0.000 & 0.004 \\
\hline 22 & Hyphoderma setigerum (Fr.) Donk & Corticiales & 0.019 & 0.000 & 0.000 \\
\hline 23 & Itersonilia perplexans Derx & Cystofilobasidiales & 0.008 & 0.000 & 0.000 \\
\hline 24 & Leucosporidium sp. & Leucosporidiales & 0.000 & 0.000 & 0.050 \\
\hline 25 & $\begin{array}{l}\text { Malassezia globosa Midgley E. Guého \& J. Guillot } 1996 \text { + M. sympodialis R.B. } \\
\text { Simmons \& E. Guého + Malassezia sp. }\end{array}$ & Malasseziales & 0.414 & 0.346 & 0.035 \\
\hline 26 & Mycena cinerella (P. Karst.) P. Karst. & Agaricales & 0.000 & 0.035 & 0.000 \\
\hline 27 & Paratritirachium sp. & Tritirachiales & 0.000 & 0.000 & 0.001 \\
\hline 28 & Peniophora piceae (Pers.) J. Erikss. & Russulales & 0.000 & 0.000 & 0.016 \\
\hline 29 & Peniophorella pubera (Fr.) P. Karst. & Corticiales & 0.003 & 0.000 & 0.004 \\
\hline 30 & $\begin{array}{l}\text { Phlebia radiata Fr. + P. rufa (Pers.) M.P. Christ. + P. tremellosa (Schrad.) } \\
\text { Nakasone \& Burds. }\end{array}$ & Corticiales & 0.000 & 0.000 & 0.176 \\
\hline 31 & Pleurotus ostreatus (Jacq.) P. Kumm. + P. cervinus (Schaeff.) P. Kumm. & Agaricales & 0.015 & 0.000 & 0.010 \\
\hline 32 & Postia rennyi (Berk. \& Broome) Rajchenb. & Polyporales & 0.011 & 0.000 & 0.000 \\
\hline 33 & Resinicium bicolor (Alb. \& Schwein.) Parmasto & Incertae sedis & 0.057 & 0.000 & 0.002 \\
\hline 34 & Russula nigricans Fr. & Russulales & 0.000 & 0.000 & 0.025 \\
\hline 35 & $\begin{array}{l}\text { Saitozyma podzolica (Babeva \& Reshetova) Xin Zhan Liu. F.Y. Bai. M. Groe- } \\
\text { new. \& Boekhout }\end{array}$ & Tremellales & 0.000 & 0.000 & 0.003 \\
\hline 36 & Sebacinales & Sebacinales & 0.000 & 0.013 & 0.002 \\
\hline 37 & Sporobolomyces roseus Kluyver \& C.B. Niel & Sporidiobolales & 0.000 & 0.000 & 0.001 \\
\hline 38 & Stereum sanguinolentum (Alb. \& Schwein.) Fr. & Russulales & 0.000 & 0.008 & 0.000 \\
\hline 39 & $\begin{array}{l}\text { Trichosporon porosum (Stautz) Middelhoven Scorzetti \& Fell + Trichosporon } \\
\text { sp. }\end{array}$ & Tremellales & 0.700 & 0.130 & 0.703 \\
\hline 40 & Wallemia sebi (Fr.) Arx & Wallemiales & 0.027 & 0.000 & 0.000 \\
\hline 41 & $\begin{array}{l}\text { Vishniacozyma carnescens (Verona \& Luchetti) Xin Zhan Liu. F.Y. Bai. M. } \\
\text { Groenew. \& Boekhout }\end{array}$ & Tremellales & 0.011 & 0.000 & 0.000 \\
\hline 42 & $\begin{array}{l}\text { Xylodon brevisetus (P. Karst.) Hjortstam \& Ryvarden }+ \text { X. detriticus (Bour- } \\
\text { dot \& Galzin) Tura Zmitr. Wasser \& Spirin }+ \text { X. flaviporus (Berk. \& M.A. } \\
\text { Curtis ex Cooke) Riebesehl \& Langer }\end{array}$ & Hymenochaetales & 0.350 & 1.165 & 0.203 \\
\hline
\end{tabular}




\begin{tabular}{|c|c|c|c|}
\hline Basidiomycota & 2.754 & 4.440 & 5.715 \\
\hline Fungi & 95.527 & 87.205 & 99.960 \\
\hline Culturable fungi & 92.311 & 85.874 & 96.896 \\
\hline Non-culturable fungi & 3.616 & 1.331 & 3.065 \\
\hline No sequence in NCBI database & 4.073 & 12.795 & 0.039 \\
\hline Pathogens & 36.630 & 37.236 & 25.046 \\
\hline Mycorrhizal fungi including & 2.061 & 0.432 & 20.157 \\
\hline Ectomycorrhizal species & 0.072 & 0.187 & 0.045 \\
\hline \multirow[t]{2}{*}{ Ericoid mycorrhiza species } & 1.989 & 0.245 & $20.112^{1}$ \\
\hline & \multicolumn{3}{|c|}{ Number } \\
\hline OTUs & 26310 & 52297 & $\begin{array}{l}185 \\
700\end{array}$ \\
\hline Taxa & 155 & 117 & 216 \\
\hline Fungal taxa & 143 & 109 & 197 \\
\hline Culturable fungi & 111 & 94 & 149 \\
\hline Non-culturable fungi & 32 & 15 & 48 \\
\hline $\begin{array}{l}\text { Zygomycota taxa } \\
\text { Ascomycota taxa }\end{array}$ & $\begin{array}{l}11 \\
71\end{array}$ & $\begin{array}{c}3 \\
72\end{array}$ & $\begin{array}{c}11 \\
105\end{array}$ \\
\hline \multirow[t]{2}{*}{ Basidiomycota taxa } & 29 & 19 & 33 \\
\hline & \multicolumn{3}{|c|}{ Indices } \\
\hline Margalef's diversity index-DMg & 15.72 & 13.35 & 17.23 \\
\hline Shannon's diversity index- H' & 0.23 & 2.54 & 2.71 \\
\hline Simpson's diversity index-D & 0.87 & 0.88 & 0.87 \\
\hline Shannon's evenness index-E & 0.05 & 0.51 & 0.51 \\
\hline Sorensen qualitative similarity index $\left(\mathrm{C}_{\mathrm{N}}\right)$ between decay class $1^{\text {st }}$ and $2^{\text {rd }}, 2^{\text {nd }}$ and $3^{\text {rd }}$ & \multicolumn{3}{|c|}{$0.5660 \quad 0.4144$} \\
\hline Sorensen qualitative similarity index $\left(\mathrm{C}_{N}\right)$ between decay class $1^{\text {st }}$ and $3^{\text {rd }}$ & \multicolumn{3}{|c|}{0.4798} \\
\hline
\end{tabular}

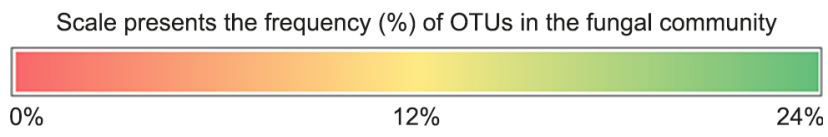

1 - An increase in ericoid mycorrhiza species was mostly attributed to an increase in frequency of Geomyces spp. which are also common in soil and alternatively they can be classified as the soil species.

by 70 taxa. Wood in the $1^{\text {st }}, 2^{\text {nd }}$ and $3^{\text {rd }}$ decay classes was colonized by 92,94 and 149 taxa of culturable fungi, respectively. The highest number of taxa of Ascomycota was in the $1^{\text {st }}$ decay class, and of Zygomycota and Basidiomycota in the $3^{\text {rd }}$ decay class.

Seventy-seven fungal taxa were common to the $1^{\text {st }}$ and $2^{\text {nd }}$ decay classes, and 51 occurred in wood of all three decay classes (Fig. 1).

The most common fungi were Ascocoryne sarcoides, Aspergillus kanagawaensis, Aureobasidium pullulans, Botryosphaeria stavensii, Botrytis cinerea, Cladosporium herbarum, C. tenuissimum, Geomyces pannorum, Lecythophora sp., Mucor abundans, Ophiostoma novo-ulmi, Penicillium spinulosum, Phialophora sp. and Scheffersomyces shehatae.

Fungi detected were: (i) typical soft rot fungi, i.e. Aspergillus spp., Cadophora spp., Chaetosphaeria spp., Lecythophora sp., Penicillium spp., Phialocephala fortinii, Phialophora sp., Phoma herbarum, Talaromyces spp.; (ii) phytopathogens potentially hazardous to trees, i.e. Armillaria spp., Bjerkandera adusta, Botryosphaeria stevensii, Cenangium acuum, Botrytis spp., Colletotrichum spp., Cosmospora sp., Daedaleopsis confragosa, Didymella macrostoma, Erysiphe alphitoides, Fusarium spp., Ganoderma applanatum, Hymenoscyphus sp., Itersonilia

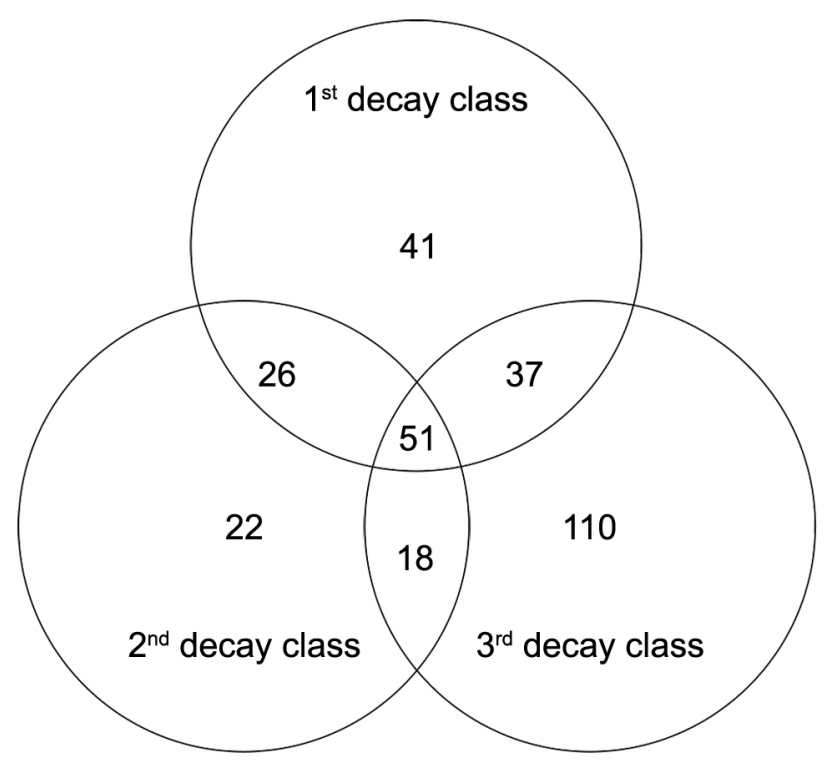

Fig. 1. Venn diagram: overlapping circles illustrate the similarities, differences, and relationships between fungal communities, with numbers of taxa shown

perplexans, Lachnellula hyalina, Nectria cinnabarina, $\mathrm{Ne}$ ocatenulostroma germanicum, Nemania serpens, Ophiostoma novo-ulmi, Pesotum piceae, Pestalotiopsis sp., 
Phaeosphaeria juncophila, Phoma herbarum, Phomopsis juglandina, Pleurostoma richardsiae, Rhizosphaera kalkhoffii, Stereum sanguinolentum, Sydowia polyspora, Taphrina purpurascens and Xenoacremonium falcatum; (iii) epiphytes and endophytes or weak, opportunistic pathogens, i.e. Alternaria spp., Aureobasidium pullulans, Babjeviella inositovora, Cladosporium spp., Epicoccum nigrum, Phialocephala spp., Phialophora sp., Postia rennyi; (iv) ectomycorrhizal Amanita rubescens, Cenococcum geophilum, Entocybe turbida, Hebeloma pamphiliense, Russula nigricans; (v) ericoid mycorrhizal Cadophora finlandica, Geomyces spp., Hyaloscypha albohyalina, Oidiodendron spp., Pseudogymnoascus spp.; (vi) crust fungi, i.e. Phlebia radiata, Peniophora piceae, Xylodon spp.; (vii) resupinate basidiomycetes, i.e. Athelia acrospora, Resinicium bicolor; (viii) saprotrophs and soil fungi, i.e. Ascoryne sarcoides, Aspergillus spp., Brachysporium nigrum, Chlorophyllum olivieri, Exophiala angulospora, Geomyces spp., Infundichalara microchona, Leptodontidium spp., Moristroma quercinum, Mucor spp., Mycena cinerella, Natantiella ligneola, Orbilia coccinella, Penicillum spp., Peniophorella pubera, Pleurotus ostreatus, Resinicium bicolor, Talaromyces spp., Umbelopsis spp., $X y$ lodon brevisetus; (ix) opportunistic human pathogens causing superficial or systemic mycoses, i.e. Cladophialophora spp., Exophiala angulospora, Lecythophora sp., Malassezia spp., Paratritirachium sp., Phoma herbarum, Rhinocladiella atrovirens, Sagenomella diversispora, Sporothrix sp.; (x) pathogens of warm- or cold-blooded animals, i.e. Geomyces spp., Sagenomella, Scytalidium sp., Malassezia spp.; (xi) ascomycetous yeasts, i.e. Babjeviella inositovora, Candida spp., Cladophialophora spp., Debaryomyces hansenii, Exophiala angulospora, Hamamotoa lignophila, Nakazawaea, Saccharomyces spp., Scheffersomyces shehatae, Sugiyamaella paludigena, Trichosporon spp.; (xii) basidiomycetous yeasts, i.e. Asterotremella sp., Cryptococcus spp., Dioszegia hungarica, Filobasidium wieringae, Genolevuria armeniaca, Hamamotoa lignophila, Leucosporidium sp., Saitozyma podzolica, Sporobolomyces roseus, Trichosporon porosum, Vishniacozyma carnescens; (xiii) antagonists of fungi, i.e. Resinicium bicolor, Trichoderma spp.; (xiv) producers of metabolites effective against pathogens, i.e. Ascocoryne sarcoides, Epicoccum nigrum, Geomyces spp., Scytalidium album; (xv) hyperparasites i.e. Asterotremella sp., Cosmospora sp., Pesotum piceae; (xvi) lichen-forming species, i.e. Fulgidea oligospora; (xvii) partners of other fungi, i.e. Infundichalara microchona which accompanies Porodaedalea pini (Brot.) Murrill.; (xviii) species rare or uncommon in nature, i.e. Babjeviella inositovora, Eucasphaeria capensis and Hymenogaster pruinatus.

The frequency of pathogens decreased with the degree of decay from $36.63 \%$ in the $1^{\text {st }}$ decay class to $25.05 \%$ in the $3^{\text {rd }}$ decay class. The frequency of mycorrhizal fungi increased with the decay continuum from $2.06 \%$ in the $1^{\text {st }}$ decay class to $20.16 \%$ in the $3^{\text {rd }}$ decay class.
Margalef's diversity index (DMg), Shannon's diversity index $\left(\mathrm{H}^{\prime}\right)$ and Simpson's diversity index (D) indicated a trend for increased diversity along the decay continuum (Table 1). The dominance of single taxa in communities resulted in small and similar values for Shannon's evenness indices (E). Shannon's evenness index (E) shows that there was more evenness in wood in the $2^{\text {nd }}$ and the $3^{\text {rd }}$ decay classes than in wood in the $1^{\text {st }}$ decay class. Berger-Parker's dominance index (d) suggests more dominance by a single species in wood in the $2^{\text {nd }}$ decay class than in wood in the $1^{\text {st }}$ or $3^{\text {rd }}$ decay classes. Sorensen qualitative similarity indices $\left(\mathrm{C}_{\mathrm{N}}\right)$ showed the fungal communities to have $41-57 \%$ similarity. The level of differences between communities are shown graphically by the heat map (Table 1).

\section{Discussion}

Until recently, deadwood was perceived as a negative element in forest ecosystems, supposedly indicating mismanagement, negligence and wastefulness (Stachura et al., 2007). Over the last decades, however, as scientific research has produced information on the functions of deadwood, the perception of deadwood in forest ecosystems has gradually changed (Merganičova et al., 2012; Gao et al., 2015). North American researchers were the first to recognize the importance of deadwood in forests (Radu, 2007). A number of publications have documented its significance for biodiversity (Ferris \& Humphrey, 1999; Humphrey et al., 2004; Müller \& Schnell, 2003; Schuck et al., 2004), nutrient cycling (Harmon et al., 1986; Krankina et al., 1999; Lexer et al., 2000; Pasinelli \& Suter, 2000), natural regeneration (Harmon \& Franklin, 1989; Mai, 1999; Ulbrichová et al., 2006; Vorčák et al., 2005, 2006; Zielonka, 2006) and other processes.

Nowadays, deadwood is of increasing interest not only to ecologists, but also to foresters and scientists. It is increasingly often recognized as an important component in the functioning of forest ecosystems (Vandekerkhove et al., 2009) and is becoming an integrated part of forest management (Marage \& Lemperiere, 2005). Deadwood has been selected as a pan-European indicator of sustainable forest management (Ministerial Conference of Protection of Forest Ecosystems (MCPFE), 2002) and is also one of 15 main indicators of biodiversity proposed by the European Environmental Agency (Humphrey et al., 2004).

The main saproxylic taxa are fungi. Fungi are the first colonizers of deadwood and begin one of the main phases of nutrient cycling (Caza, 1993). Specialized wood-inhabiting fungi decompose organic molecules of wood, by production of wood-degrading 
enzymes, and thus release nutrients for plants. The various processes that they employ to degrade wood components result, directly or indirectly, in further decay of wood, which becomes colonized by unspecialized species, next in the fungal succession (Hammel et al., 2002; Kwaśna et al., 2016a, b; 2017). The pattern of fungal distribution can provide specific information on the significance of fungi in the functioning and stability of the ecosystem (Kubartová et al., 2012).

The present study on fungal colonization of log deadwood of $Q$. petraea was made in awareness that bark and heartwood are generally more resistant than sapwood to fungal colonization and decomposition, and that separate analysis of each would be advisable. It was decided, however, to pool heartwood and sapwood for mycological analysis because (i) no important differences between the according to van der Wal et al. (2017) there was no bigger difference in structure of fungal communities from heartwood and sapwood of Quercus rubra L. had been found (van der Wal et al., 2017), and (ii) it was often difficult to separate heartwood from sapwood in the $3^{\text {rd }}$ decay class.

In the six 3-30-year-old $Q$. petraea logs there were 264307 different OTUs, which is 127 -fold more than the 2080 OTUs found in nine samples of 3-year-old Q. rubra logs using high-throughput sequencing (by van der Wal et al., 2017), but less than the 687662 and 1861279 OTUs found in 20 samples of broadleaved trees from two locations in neotropical Atlantic forests using massively parallel sequencing (Vaz et al., 2017). There were also 277 fungal taxa, which is more than the 141 taxa detected in deadwood of Quercus robur L. using the classical method based on isolation and microscopic analysis (Kwaśna et al., 2016b), the 100 taxa detected in neotropical Atlantic forests (Vaz et al., 2017), and the 15 species detected in Q. rubra logs (by van der Wal et al., 2017). In two other tree species, beech and Scots pine, respectively 96 and 138 fungal taxa were detected by isolation and microscopic analysis (Fukasawa et al., 2009; Kwaśna et al., 2016b). The impressive total of 277 taxa was recorded despite the presence of a microbial barrier and selection for specialized rotting fungi, which can decompose the highly resistant oak wood containing such organic compounds as tannins and suberin (Vane et al., 2006; van der Wal et al., 2017).

There was a significant positive relationship between wood decay class and fungal diversity. This agrees with observations of Rajala et al. (2011, 2015) and van der Wal et al. $(2015,2016,2017)$ but not with Kwaśna et al. (2017), probably because of the different techniques used for fungal detection. According to Rajala et al. (2015), fungal diversity is inversely related to wood density and $\mathrm{C} / \mathrm{N}$ ratio (which decrease along the decay continuum) and directly related to moisture and the lignin content of wood (which increase along the decay continuum). Competition, succession or complementarity among fungi may, however, contribute to the final diversity as well as to the decay rate. Thus, the exact mechanisms for positive or negative diversity-wood decay relationships in species-rich communities remain largely unknown and are challenging to investigate.

Ascomycota were dominant, particularly in wood of the $1^{\text {st }}$ decay class where their frequency increased to over $89 \%$ of all fungi detected. Dominance of Ascomycota in deadwood was also observed by Vaz et al. (2017). Many Ascomycota taxa detected were soft rot fungi, meaning that they can break down cellulose and hemi-cellulose, but not harder structural substances such as lignin (Mäkelä et al., 2015). The high abundance of Ascomycota in oak wood was expected since they are more frequent in wood of deciduous than coniferous trees (Gutowski et al., 2004; Kwaśna et al., 2016b). Deciduous trees are more susceptible than coniferous trees to colonization by Ascomycota because of their higher content of cellulose and hemicellulose, but not of lignin, which is in greater amounts in conifers. Coniferous species also contain resins, which limit fungal colonization (McKnight \& Mullins, 1981; Macdonald et al., 2016). The abundance and wide distribution of Ascomycota may also be explained by their intensive production of easily-dispersed spores.

Ascomycota were still dominant in the $2^{\text {nd }}$ and $3^{\text {rd }}$ decay classes (with high frequency of $81-87 \%$ ) and associated with only a small increase of Basidiomycota. The soft rot phase caused by Ascomycota in oak deadwood is therefore long and progresses slowly, the fungi not being replaced by more aggressive but less frequent wood-decaying Basidiomycota (Vane et al., 2006). In nature, soft rot usually occurs when wet and dry seasons alternate. It is limited to surface layers of wood $(2-4 \mathrm{~mm})$ in periods of higher humidity. In a drought the decomposed wood crumbles and the deeper layers are exposed to further colonization and decomposition. High moisture in wood inhibits the growth of wood-decaying Basidiomycota. The lower concentrations of toxic carbon monoxide in the exposed layers of wood favour colonization by fungi (Eaton \& Hale, 1993).

Fukasawa et al. (2011) reported that soft rot taxa are secondary colonizers, present during the final stages of wood decomposition. In the present study, however, greatest abundance of Ascomycota occurred in the early stages of wood decay, although fungal diversity increased substantially along the decay continuum. This is consistent in part (abundance but not diversity on/in oak deadwood) with earlier studies (Kwaśna et al., 2016 b). Less abundance associated with greater diversity may result from a changed structure, physical characters and chemical 
composition of the wood and the location and position of the logs.

Logs of Q. petraea were shown to be a habitat for pathogens potentially dangerous to trees and hazardous to forest health. They are often pathogens of foliage, stems, trunks and roots, with cosmopolitan distribution. Armillaria spp., Bjerkandera adusta, Daedaleopsis confragosa, Ganoderma applanatum, Hyphoderma setigerum, Resinicium bicolor and Stereum sanguinolentum can cause root rot, white rot or red heart rot on living or injured conifers and deciduous trees (Schmidt, 2006). Botryosphaeria stevensii, Lachnellula hyalina, Nectria cinnabarina, Phomopsis juglandina and Xenoacremonium falcatum have been frequently associated with dieback and cankers in oak, larch, walnut and other deciduous trees (Alves et al., 2004; Anagnostakis, 2007; Lombard, et al., 2014). Cenangium acuum, Neocatenulostroma germanicum, Rhizosphaera kalkhoffii and Sydowia polyspora colonize pine and spruce needles and cause needle blight or needle necrosis and cast (Skilling \& Walla, 1986; Markovskaja et al., 2016). The genus Colletotrichum contains important anthracnose pathogens (Damm et al., 2010). Erysiphe alphitoides causes powdery mildew on oak trees. The Fusarium spp. detected can cause damping-off, wilting of the foliage, necrosis and cankers of the bark (Gerlach \& Nirenberg, 1982). Hymenoscyphus causes ash-dieback (Kowalski \& Holdenrieder, 2009), Itersonilia perplexans causes flower-petal blight and cankers (Boekhout et al., 1991) and Pestalotiopsis sp. causes diseases of foliage, stems and roots (Maharachchikumbura et al., 2014). Phaeosphaeria species are pathogens of cereals, grasses, sedges, rushes, and grass-like plants. Pleurostoma richardsiae is a lesser known vascular pathogen (Carlucci et al., 2013). Species of Alternaria, Athelia, Aureobasidium pullulans, Cladosporium spp., Botrytis spp., Brachysporium nigrum, Epicoccum nigrum and Phoma herbarum are facultative parasites of plants (including crops) and of lichens (Esslinger, 2009). The abundance of pathogens decreased with the decay continuum, in agreement with van der Wal et al. (2017). Some pathogens, i.e. Botryosphaeria stevensii, Botrytis spp., Colletotrichum spp., Fusarium spp., Hymenoscyphus sp., Nectria cinnabarina, Neocatenulostroma germanicum, Pesotum piceae, Phoma herbarum, Phomopsis juglandina and Sydowia polyspora, had almost disappeared from wood in the $3^{\text {rd }}$ decay class.

Ophiostoma novo-ulmi was among the most abundant fungi and dominant in all three decay classes. It causes the current Dutch elm disease (DED) pandemics and so far there have been no other records of $O$. novo-ulmi in oak deadwood. However, a high affinity of O. novo-ulmi to O. quercus (Georgev.) Nannf (expected on oak wood) has been noted (Harrington et al., 2001), which may explain its occurrence on the oak deadwood.
Armillaria gallica and A. cepistipes were most abundant in wood in the $2^{\text {nd }}$ decay class, consistent with earlier findings (Kwaśna et al., 2016b). Both species are known as opportunistic parasites in weakened or stressed trees (particularly A. gallica in oak). They may also act as saprotrophs, however, being able to decompose effectively plant residues containing lignin.

Each stage of decomposition seems to favour colonization by different pathogens. Those associated with dieback and cankers, e.g. Botryosphaeria stevensii, Fusarium spp., Nectria cinnabarina and Phomopsis juglandina, colonize wood at the early stages of decomposition, and those associated with wilt and necrosis, e.g. Ophiostoma novo-ulmi, at the advanced stage. This results from specific adaptations for utilizing different qualities of deadwood, which differ according to tree species, wood type (heartwood, sapwood or bark), sample size and decay rate (Abrego \& Salcedo, 2013). Although the frequency of pathogens decreased with increasing decay, the continuous presence of most pathogen species in deadwood represents a health risk for the forest, fungal diversity and fungal species conservation.

An increase in fungal diversity and a small decrease in abundance of Ascomycota as decay advanced were associated with only a small increase in the abundance of Basidiomycota, including pathogens, mycorrhizal species, white rot species and yeasts. This agrees with Rajala et al. (2011, 2012), Kubartová et al. (2012) and Ovaskainen et al. (2013) but not with Crawford et al. (1990), Lumley et al. (2001), Fukasawa et al. $(2009,2010)$ or Kim et al. (2009); the differences seem to result from inconsistency of methodologies used. Effects of position of the sampled logs on fungal diversity must, however, also be considered. In this study the sampled logs were lying horizontally on the ground. They were not only exposed to the local microclimate but also to colonization via air, by wind, or from soil (common for Ascomycota) rather than from roots (possible for pathogenic or mycorrhizal Basidiomycota).

Basidiomycota occurred much less frequently than Ascomycota at each stage in the decomposition of log deadwood of $Q$. petraea. This is consistent with earlier findings (Kwaśna et al., 2016b). Ectomycorrhizal species were rare. There were only single records of the bracket fungi, Daedaleopsis confragosa and Ganoderma applanatum. Only a few crust and resupinate $\mathrm{Ba}-$ sidiomycota, Athelia sp., Phlebia radiata, Peniophorella, sp. and Resinicium bicolor, detected in this study were also found among 23 species from 3-year-old Q. rubra logs (vanstudied by van der Wal et al., 2017). However, the low similarity of mycobiota between the two studies may be explained by the different primer (fITS9) and methodology (high-throughput sequencing) used by van der Wal et al. (2017). 
Although ectomycorrhizal fungi were most abundant in the $2^{\text {nd }}$ decay class, colonization had already started in the earlier stage, in agreement with Renvall (1995), Rajala et al. $(2012,2015)$ and Ottosson et al. (2014). Wood-colonizing mycorrhizal species, apart from their primary function of translocating nutrients from soil to plant roots, also the ability to decompose wood (Lindahl \& Tunlid, 2015). Some species even tend to produce fruit bodies more often in wood than in soil (Stokland et al., 2012). Conversely, other wood-decay fungi (in particular cord-forming Basidiomycota) are able to grow out of the wood and forage for new resources in soil (Boddy, 1993, 1999). An increase in mycorrhizal taxa in the $3^{\text {rd }}$ decay class was mostly attributable to an increase in frequency of Geomyces spp. Apart from being ericoid mycorrhiza species, Geomyces spp. are also common in soil and can be classified as soil species.

Many saprotrophs detected, e.g. Capronia pilosella, Dematioscypha spp., Hyaloscypha albohyalina, Hypoxylon rubiginosum, Lecythophora sp., Moristroma quercinum, Natantiella ligneola, Nemania serpens, Oidiodendron spp., Orbilia spp., Paratritirachium sp., Penicillium spp., Peniophorella pubera, Phlebia spp., Pleurotus ostreatus, Postia rennyi, Sporothrix sp., Talaromyces spp., Trichoderma spp., Wallemia sebi and Xylodon spp., are known from decorticated and highly decayed wood of Quercus and other deciduous tree species or from soil (Domsch et al, 1980; Müller et al., 1987; Wojewoda, 2003). Others; Réblová \& Stepánek (2009). Other are known from conifer habitats: coniferous tree roots (Archaeorhizomyces finlayi), cones (Baeospora sp.), needles and coniferous litter (Mycena cinerella) or coniferous wood (Peniophora piceae) (Tyler, 1991; Hutchinson et al., 2012).

Wood in the $3^{\text {rd }}$ decay class became a habitat for many soil fungi. Their frequency had been increasing with the decay continuum. Decay eventually results in the dead wood constituents becoming soil, in which wood and soil-inhabiting fungal communities converge. Wood-soil and soil-wood transfers have important implications for fungal community dynamics, recycling of forest nutrients, activity of mycorrhiza, relocation of resources and overall nutrient fluxes in forest ecosystems (Philpott et al., 2014; Mäkipää et al., 2017).

Soil fungi were represented mainly by Zygomycota and ascomycetous Aspergillus spp., Geomyces spp., Penicillium spp., Sporothrix sp. and Trichoderma spp. In various habitats they often need to employ different ecophysiological strategies adapted to the characteristics of the substrate (Watkinson et al., 2006). Increase in abundance of Zygomycota, which generally do not have any cellulolytic or ligninolytic abilities and are secondary colonizers of substrata rich in simple carbohydrates (Hudson, 1968; Nilsson, 1973; Osono \& Takeda, 2001), in the strongly decayed wood, was presumably made possible by earlier decomposition of wood by other fungi. Geomyces species are usually particularly common in soils of temperate and high-latitude ecosystems (Kirk et al., 2008). They are capable of withstanding, and thriving in, cold, low-nutrient environments (Hayes, 2012)

Soil can be a source of inoculum for fungal colonization and decomposition of deadwood (van der Wal et al., 2007). Polyphenolic materials from vegetation diffusing into the deadwood often affect fungal abundance and diversity. Logs sampled for the present study were lying on a poor soil (ironrich podzolic soil formed on sand), which supported growth of only a few plant species, mostly grasses. However, organic matter composed of the remains of vegetation presumably affected the abundance and diversity of fungi, first in the soil and later in lying deadwood. More information is needed on the effects of soil properties and vegetation on wood decomposition by fungi.

Among antagonists, the genus Trichoderma was not as abundant as has been suggested by studies based on the classical method isolation of fungi on artificial media and microscopic analysis (Kwaśna et al., 2016b). Their least abundance in the primary stage of decay can be explained by their lack of enzymes that decompose lignin, which is still present in structures during early colonization (lamellae of cells and outer cell walls) (Eaton \& Hale, 1993; Fukasawa et al., 2009). Before utilizing cellulose, Trichoderma requires prior decomposition of lignin by other fungi (Schwarze et al., 2000; Schmidt, 2006).

Among hyperparasitic fungi detected, Asterotremella sp. is known to grow on agarics (Prillinger et al., 2007) and Cosmospora sp. on polypores and xylariaceous fungi (Gräfenhan et al., 2011). Pesotum piceae grows on mats of Ceratocystis fagacearum (Bretz) J. Hunt. (Ruetze \& Parameswaran, 1984); thus, by reducing the chance of contamination of insect vectors with spores of $C$. fagacearum, P. piceae is likely to contribute to the low efficiency of oak wilt transmission.

The character and size of the wood samples analysed may have affected fungal diversity. The samples originated from logs lying horizontally on the ground, and were large $(60 \times 20 \times 20 \mathrm{~cm})$. Their choice was dictated by earlier findings (Lindhe et al., 2004), in which fungal diversity (based on fruit-body inventory) was found to be greater on logs than on stumps and increased with the size of sample. It seems that that larger wood pieces were able to maintain the more stable microclimatic conditions required by some specialized species (Heilmann-Clausen \& Christensen, 2004; Kwaśna et al., 2017; van der Wal et al., 2017). There was much variation among the large numbers of taxa favouring each of the different decay classes. The succession of fungi along the wood decay continuum is in agreement with other 
studies (Renvall, 1995; Rajala et al., 2012; Ottosson et al., 2014).

Fungal communities in the oak deadwood studied here, in the continental climate of Europe, did not include as many Sordariomycetes as observed in deadwood in tropical broad-leaved forests (Vaz et al., 2017). This class was represented here by the very frequent Lecythophora sp. and Ophiostoma novo-ulmi, and non-frequent members of Calosphaeriales, Chaetosphaeriales, Diaportales, Hypocreales, Sordariales, Trichosphaeriales and Xylariales. Leotiomycetes (Ascomycota) was the most frequently represented class; it included Helotiales (13 taxa) + Incertae sedis (five taxa: Geomyces, Natantiella, Oidiodendron spp., Pseudogymnoascus spp. and Scytalidium album). The other more common orders were Saccharomycetales in class Saccharomycetes (seven taxa), Chaetothyriales (six taxa) and Eurotiales (five taxa) in Eurotiomycetes class Eurotiomycetes, Capnodiales (two taxa) and Pleosporales (five taxa) in class Dothideomycetes. Four species of Ascomycota, i.e. Ascocoryne sarcoides, Leptodontidium trabinellum, Moristroma quercinum and Pestalotiopsis sp., were common to oak and tropical broad-leaved deadwood (Vaz et al., 2017).

The wood-decaying Basidiomycota included mostly white rot fungi. The majority of taxa (i.e. Agaricales, Atheliales, Corticiales, Hymenochaetales, Polyporales, Russulales, Tremellales) are Agaricomycetes. There was only one taxon of brown rot fungus, Athelia acrospora. Van der Wahl et al., (2017) found, however, Athelia decipiens (Höhn. \& Litsch.), Daedalea quercina (L.) Pers. and Serpula himantoides (Fr.) P. Karst on deadwood of $Q$. rubra.

The majority of fungi $(90 \%)$ were identified to genus or species level. Application of the Illumina technique, which achieves sequencing by synthesis, and identification based on probability, therefore ensured a high level of accuracy and reliability. Classification of fungi was more effective than in other studies in which 454 sequences resulted in $40 \%$ of sequences unidentified even to genus level (Ovaskainen et al., 2010; Kubartová et al., 2012).

Unexpectedly, DNA of Solanum pennellii, a wild green-fruited tomato with extreme stress tolerance and unusual morphology, endemic to Andean regions in South America, was detected in wood at the $3^{\text {rd }}$ decay class. The most reasonable explanation for this finding is a taxonomic mistake made by the depositor.

Conclusions: Coarse, woody debris of oak is host to abundant and diverse mycobiota. Density and diversity of fungi increase with the decay continuum. Oak logs can be a habitat for phytopathogens potentially hazardous for forest health. Different phytopathogens favour wood in different decay classes. Abundance of pathogens decreases, and of ericoid mycorrhizal (or soil) species increases with the decay continuum. Maintaining different types of deadwood increases fungal diversity, but also the risk of diseases.

\section{References}

Abrego N \& Salcedo I (2013) Variety of woody debris as the factor influencing wood inhabiting fungal richness and assemblages: Is it a question of quantity or quality? Forest Ecology and Management 291: 377-385. doi:org/10.1016/j.foreCo.2012.11.025.

Allen RB, Buchanan PK, Clinton PW \& Cone AJ (2000) Composition and diversity of fungi on decaying logs in a New Zealand temperate beech (Nothofagus) forest. Canadian Journal of Forest Research 30: 1025-1033.

Allmér J, Vasiliauskas R, Ihrmark K, Stenlid J \& Dahlberg A (2006) Wood-inhabiting fungal communities in woody debris of Norway spruce (Picea abies (L.) Karst.), as reflected by sporocarps, mycelial isolations and T-RFLP identification. FEMS Microbiology Ecology 55: 57-67.

Alves A, Correia A, Luque J \& Phillips A (2004) Botryosphaeria corticola, sp. nov. on Quercus species, with notes and description of Botryosphaeria stevensii and its anamorph, Diplodia mutila. Mycologia 96: 598-613.

Anagnostakis SL (2007) Diaporthe eres (Phomopsis oblonga) as a pathogen of butternut (Juglans cinerea) in Connecticut. Plant Disease 91: 1198. doi:org/10.1094/PDIS-91-9-1198C.

Andersson L \& Hytteborn H (1991) Bryophytes and decaying wood - a comparison between managed and natural forest. Ecography 14: 121-130.

Angelstam PK, Butler R, Lazdinis M, Mikusinski G \& Roberge JM (2003) Habitat thresholds for focal species at multiple scales and forest biodiversity conservation - dead wood as an example. Annales Zoologici Fennici 40: 473-482.

Błońska E, Kacprzyk M \& Spólnik A (2017) Effect of deadwood of different tree species in various stages of decomposition on biochemical soil properties and carbon storage. Ecological Research 32: 193-203.

Boddy L (1993) Saprotrophic cord-forming fungi: warfare strategies and other ecological aspects. Mycological Research 97: 641-655.

Boddy L (1999) Saprotrophic cord-forming fungi: meeting the challenge of heterogeneous environments. Mycologia 91: 13-32.

Boddy L, Frankland J \& van West P (2008) Ecology of saprotrophic basidiomycetes. Academic Press, London. 
Boekhout T, Poot G, Hackman P \& Steensma HY (1991) Genomic characteristics of strains of Itersonilia: Taxonomic consequences and life cycle. Canadian Journal of Microbiology 37: 188-194. doi:org/10.1139/m91-029.

Carlucci A, Raimondo ML, Cibelli F, Phillips AJL \& Lops F (2013) Pleurostomophora richardsiae, Neofusicoccum parvum and Phaeoacremonium aleophilum associated with a decline of olives in southern Italy. Phytopathologia Mediterranea 52: 517-527. doi:10.14601/Phytopathol_Mediterr-13526.

Caza CL (1993) Woody debris in the forests of British Columbia: a review of the literature and current research. B.C. Ministry of Forests Land Management Report No. 78. Victoria, British Columbia.

Cole JR, Wang Q, Fish JA, Chai B, McGarrell DM, Sun Y, Brown CT, Porras-Alfaro A, Kuske CR, \& Tiedje JM (2014) Ribosomal Database Project: data and tools for high throughput rRNA analysis. Nucleic Acids Research, 42: D633-D642.

Crawford RH, Carpenter SE \& Harmon ME (1990) Communities of filamentous fungi and yeast in decomposing logs of Pseudotsuga menziesii. Mycologia 82: 759-765.

Czerepko J, Boczoń A, Cieśla A, Forycka A, Ksepko M, Obidziński A, Paluch R, Rodziewicz A, Różański W, Sokołowski K, Szwed W \& Wróbel M (2008) Stan różnorodności biologicznej lasów $\mathrm{w}$ Polsce na podstawie powierzchni obserwacyjnych monitoringu. Instytut Badawczy Leśnictwa, Sękocin Stary.

Damm U, Baroncelli R, Cai L, Kubo Y, O'Connell R, Weir B, Yoshino K \& Cannon PF (2010) Colletotrichum: species, ecology and interactions. IMA Fungus 1: 161-165.

DeMaynadier PG \& Hunter ML (1995) The relationship between forest management and amphibian ecology: a review of the North American literature. Environmental Review 3: 230-261.

Domsch KH, Gams W \& Anderson TH (1980) Compendium of soil fungi. Academic Press, London, U.K.

Eaton RA \& Hale MDC (1993) Wood: decay, pests and protection. Chapman \& Hall, London, UK.

Esslinger TL (2009) A cumulative checklist for the lichen-forming, lichenicolous and allied fungi of the continental United States and Canada. North Dakota State University.

Fauteux D, Imbeau L, Drapeau P\& Mazerolle MJ (2012) Small mammal responses to coarse woody debris distribution at different spatial scales in managed and unmanaged forests. Forest Ecology and Management 266: 194-205.

Ferris R \& Humphrey JW (1999) A review of potential biodiversity indicators for application in British forests. Forestry 72: 313-328
Ferris R, Peace AJ \& Newton AC (2000) Macrofungal communities of lowland Scots pine (Pinus sylvestris L.) and Norway Spruce (Picea abies (L.) Karsten) plantations in England: Relationships with site factors and stand structure. Forest Ecology and Management 131: 255-267.

Fischer AL, Moncalvo JM, Klironomos JN \& Malcolm JR (2012) Fruiting body and molecular rDNA sampling of fungi in woody debris from logged and unlogged boreal forests in northeastern Ontario. Ecoscience 19: 374-390.

Forest Europe (2011) State of Europe's forests 2011. Status and trends in sustainable forest management in Europe.

Fukasawa Y, Osono T \& Takeda H (2009) Microfungus communities of Japanese beech logs at different stages of decay in a cool temperate deciduous forest. Canadian Journal of Forest Research 39: 1606-1614. doi:org/10.1139/X09-080.

Fukasawa Y, Osono T \& Takeda H (2010) Beech log decomposition by wood-inhabiting fungi in a cool temperate forest floor: a quantitative analysis focused on the decay activity of a dominant basidiomycete Omphalotus guepiniformis. Ecological Research 25: 959-966.

Fukasawa Y, Osono T \& Takeda H (2011) Wood decomposing abilities of diverse lignicolous fungi on non-decayed and decayed beech wood. Mycologia 103: 474-482. doi:org/10.3852/10-246.

Gao T, Nielsen AB \& Hedblom M (2015) Reviewing the strength of evidence of biodiversity indicators for forest ecosystems in Europe. Ecological Indicators 57: 420-434.

Gerlach W \& Nirenberg H (1982) The genus Fusarium - a pictorial atlas. Mitteilungen aus der Biologischen Bundesanstalt für Land- und Forstwirtschaft 209: 1-406.

Gräfenhan T, Schroers HJ, Nirenberg HI \& Seifert KA (2011) An overview of the taxonomy, phylogeny, and typification of nectriaceous fungi in Cosmospora, Acremonium, Fusarium, Stilbella, and Volutella. Studies in Mycology 68: 79-113. doi:10.3114/ sim.2011.68.04.

Grove SJ (2002) Saproxylic insect ecology and the sustainable management of forests. Annual Review of Ecology and Systematics 33: 1-23.

Gutowski JM, Bobiec A, Pawlaczyk P \& Zub KB (2004) Drugie życie drzew. WWF, Warszawa-Hajnówka.

Hahn K \& Christensen M (2005) Dead wood in European forest reserves - a reference for forest management. EFI Proceedings 51: 181-191.

Hammel KE, Kapich AN, Jensen KA \& Ryan ZC (2002) Reactive oxygen species as agents of wood decay by fungi. Enzyme and Microbial Technology 30: $445-453$. 
Harmon ME \& Franklin JF (1989) Tree seedlings on logs in Picea-Tsuga forests of Oregon and Washington. Ecology 70: 48-59.

Harmon ME, Franklin JF, Swanson FJ, Sollins P, Gregory SV, Lattin JD, Anderson NH, Cline SP, Aumen NG, Sedell JR Lienkaemper GW, Cromack K \& Cummins KW (2004) Ecology of coarse woody debris in temperate ecosystems. Advances in Ecological Research 34: 59-234.

Harmon ME, Franklin JF, Swanson FJ, Sollins P, Gregory SV, Lattin JD, Anderson NH, Cline SP, Aumen NG, Sedell JR Lienkaemper GW, Cromack K \& Cummins KW (1986) Ecology of coarse woody debris in temperate ecosystems. Advances in Ecological Research 15: 133-302.

Harrington TC, McNew D, Steimel J, Hofstra D \& Farrell R (2001) Phylogeny and taxonomy of the Ophiostoma piceae complex and the dutch elm disease fungi. Mycologia 93: 111-136.

Hayes MA (2012) The Geomyces fungi: ecology and distribution. BioScience 62: 819-823.

Heilmann-Clausen J \& Christensen M (2004) Does size matter? On the importance of various dead wood fractions for fungal diversity in Danish beech forests. Forest Ecology and Management 201: 105-117. doi:10.1016/j.foreco.2004.07.010.

Holub SM, Spears JD \& Lajtha KA (2001) Reanalysis of nutrient dynamics in coniferous coarse woody debris. Canadian Journal of Forest Research 31: 1894-1902.

Hoppe B, Kahl T, Karasch P, Wubet T, Bauhus J, Buscot F \& Krüger D (2014) Network analysis reveals ecological links between N-fixing bacteria and wood-decaying fungi PLoS One 9: e88141. doi:10.1371/journal. pone. 0088141.

Hudson HJ (1968) The ecology of fungi on plant remains above the soil. New Phytologist 67: 837874.

Humphrey JW, Sippola AL, Lemperiere G, Dodelin B, Alexander KNA \& Butler JE (2004) Deadwood as an indicator of biodiversity in European forests: from theory to operational guidance. EFI Proceedings 51: 193-206.

Hunter ML Jr (1990) Wildlife, forests and forestry: principles of managing forests for biological diversity. Prentice-Hall, Englewood Cliffs, New Jersey, USA.

Hutchinson LJ, Kropp BR \& Hausner G (2012) Baeospora occidentalis, a new snowbank agaric from western North America. Mycoscience 53: 139-143. doi:10.1007/s10267-011-0142-3.

Ihrmark K, Bödeker IT, Cruz-Martinez K, Friberg H, Kubartova A, Schenck J, Strid Y, Stenlid J, Brandström-Durling M, Clemmensen KE \& Lindahl BD (2012) New primers to amplify the fungal ITS2 region - evaluation by 454 -sequencing of artifi- cial and natural communities. FEMS Microbiology Ecology 82: 666-677. doi:10.1111/j.15746941.2012.01437.x.

Kappes H, Jabin M, Kulfan J, Zach P \& Topp W (2009) Spatial patterns of litter dwelling taxa in relation to the amount of coarse woody debris in European temperate deciduous forests. Forest Ecology and Management 257: 1255-1260.

Kebli H, Drouin P, Brais S \& Kernaghan G (2011) Species composition of saproxylic fungal communities on decaying logs in the boreal forest. Microbial Ecology 61: 898-910.

Kebli H, Brais S, Kernaghan G \& Drouin P (2012) Impact of harvesting intensity on wood-inhabiting fungi in boreal aspen forests of Eastern Canada. Forest Ecology and Management 279: 45-54.

Kim GH, Lim YW, Choi YS, Kim MJ \& Kim JJ (2009) Primary and secondary decay fungi on exposed pine tree logs in the forest. Holzforschung 63: 633-638. doi:10.1515/HF.2009.099.

Kirk PM, Cannon PF, Minter DW \& Stalpers JA (2008) Dictionary of the fungi. CAB International. UK.

Klein D, Höllerl S, Blaschke M \& Schulz C (2013) The contribution of managed and unmanaged forests to climate change mitigation-A model approach at stand level for the main tree species in Bavaria. Forests 4: 43-69.

Kowalski T \& Holdenrieder O (2009) The teleomorph of Chalara fraxinea, the causal agent of ash dieback. Forest Pathology 39: 304-308. doi:10.1111/ j.1439-0329.2008.00589.x.

Krankina ON, Harmon ME \& Griazkin AV (1999) Nutrient stores and dynamics of woody detritus in a boreal forest: modeling potential implications at the stand level. Canadian Journal of Forest Research 29: 20-32.

Kubartová A, Ottosson E, Dahlberg A \& Stenlid J (2012) Patterns of fungal communities among and within decaying logs, revealed by 454 sequencing. Molecular Ecology 21: 4514-4532. doi:10.1111/j.1365-294X.2012.05723.x.

Küffer N \& Senn-Irlet B (2005) Influence of forest management on the species richness and composition of wood-inhabiting basidiomycetes in Swiss forests. Biodiversity and Conservation 14: 2419-2435.

Kulhánková A, Béguiristain T, Moukoumi, Berthelin J \& Ranger J (2006) Spatial and temporal diversity of wood decomposer communities in different forest stands, determined by ITS rDNA targeted TGGE. Annals of Forest Science 63: 547-556.

Kwaśna H, Łakomy P \& Gornowicz R (2016a) Grzyby saproksyliczne $w$ resztkach pozrębowych sosny zwyczajnej. Sylwan 160: 355-364.

Kwaśna H, Mazur A, Kuźmiński R, Jaszczak R, Turski M, Behnke-Borowczyk J, Adamowicz K \& 
Łakomy P (2017) Abundance and diversity of wood-decay fungi in managed and unmanaged stands in a Scots pine forest in western Poland. Forest Ecology and Management 400: 438-446. doi:org/10.1016/j.foreco.2017.04.023.

Kwaśna $H$, Mazur A, Łabędzki A, Kuźmiński R \& Łakomy P (2016b) Communities of fungi in decomposed wood of oak and pine. Leśne Prace Badawcze 77: 261-275. doi:10.1515-frp-2016-0028.

Lexer MJ, Lexer W \& Hasenauer H (2000) The use of forest models for biodiversity assessments at the stand level. Investing Agrar Sist Recur For Fuera de Serie $\mathrm{n}^{\circ} 1$, pp. 297-316.

Lindahl BD \& Tunlid A (2015) Ectomycorrhizal fungi-potential organic matter decomposers, yet not saprotrophs. New Phytologist 205: 1443-1447. doi:10.1111/nph.13201.

Lindhe A, Åsenblad N \& Toresson HG (2004) Cut logs and high stumps of spruce, birch, aspen and oak-nine years of saproxylic fungi succession. Biological Conservation 119: 443-454. doi:10.1016/j.biocon.2004.01.005.

Lindner DL, Burdsall Jr. HH \& Stanosz GR (2006) Species diversity of polyporoid and corticioid fungi in northern hardwood forests with differing management histories. Mycologia 98: 195-217.

Lindner DL, Vasaitis R, Kubartová A, Allmér J, Johannesson H, Banik MT \& Stenlid J (2011) Initial fungal colonizer affects mass loss and fungal community development in Picea abies logs 6yr after inoculation. Fungal Ecology 4: 449-460.

Lombard L, van der Merwe NA, Groenewald JZ \& Crous PW (2014) Generic concepts in Nectriaceae. Studies in Mycology 80: 189-245. doi:10.1016/j. simyco.2014.12.002.

Lumley TC, Gignac LD \& Currah RS (2001) Microfungus communities of white spruce and trembling aspen logs at different stages of decay in disturbed and undisturbed sites in the boreal mixed wood region of Alberta. Canadian Journal of Botany 79: 76-92. doi:org/10.1139/b00-135.

Mac Nally R, Parkinson A, Horrocks G, Conole L \& Tzaros C (2001) Relationships between terrestrial vertebrate diversity, abundance and availability of coarse woody debris on south-eastern Australian floodplains. Biological Conservation 99: 191-205.

Macdonald J, Goacher RE, Abou-Zaid M \& Master ER (2016) Comparative analysis of lignin peroxidase and manganese peroxidase activity on coniferous and deciduous wood using ToF-SIMS. Applied Microbiology and Biotechnology 100: 8013-80-20.

Magurran AE (1988) Ecological diversity and its measurement. Princeton University Press. USA.

Maharachchikumbura SSN, Hyde KD, Groenewald JZ, Xu J \& Crous PW (2014) Pestalotiopsis revisited.
Studies in Mycology 79: 121-186. doi:10.1016/j. simyco.2014.09.005.

Mai W (1999) Über Ammenstäemme im Gebirgswald. LWF 18: 18-20.

Mäkelä MR, Marinović M, Nousiainen P, Liwanag AJ, Benoit I, Sipilä J, Hatakka A, de Vries RP \& Hildén KS (2015) Aromatic metabolism of filamentous fungi in relation to the presence of aromatic compounds in plant biomass. Advances in Applied Microbiology 91: 63-137.

Mäkipää R, Rajala T, Schigel D, Rinne KT, Pennanen T, Abrego N \& Ovaskainen O (2017) Interactions between soil- and dead wood-inhabiting fungal communities during the decay of Norway spruce logs. International Society for Microbial Ecology Journal 11: 1964-1974. doi:10.1038/ismej.2017.57.

Marage D \& Lemperiere G (2005) The management of snags: A comparison in managed and unmanaged ancient forests of the Southern French Alps. Annals of Forest Science 62: 135-142.

Markovskaja S, Kačergius A, Davydenko K \& Fraser S (2016) First record of Neocatenulostroma germanicum on pines in Lithuania and Ukraine and its co-occurrence with Dothistroma spp. and other pathogens. Forest Pathology 46: 522-533. doi:10.1111/efp.12308.

Maser C \& Trappe JM (1984) The seen and unseen world of the fallen tree. General Technical Reports PNW-164. Department of Agriculture, Forest Service, Pacific Northwest Forest and Range Experiment Station, Portland, USA.

McComb WC \& Lindenmayer D (1999) Dying, dead, and down trees: Maintaining biodiversity in forest ecosystems (ed. by ML Hunter) Cambridge University Press, Cambridge, UK, pp. 335-372.

McKnight TE \& Mullins EJ (1981) Canadian woods, their properties and uses. The University of Toronto Press, Canada.

MCPFE (2002) Improved Pan-European indicators for sustainable forest management as adopted by the MCPFE Expert Level Meeting 2002. Vienna, Austria.

Merganičova K, Merganič J, Svoboda M, Bače R \& Šebeň V (2012) Deadwood in forest ecosystems: In forest ecosystems - more than just trees (ed. by JA Blanco) Intech Open, London, UK, pp. 81-08. doi:10.5772/1127.

Müller E, Petrini O, Fisher PJ, Samuels GJ \& Rossman AY (1987) Taxonomy and anamorphs of the Herpotrichiellaceae with notes on generic synonymy. Transactions of the British Mycological Society 88 : $63-74$.

Müller J \& Schnell A (2003) Was lernen wir, wenn wir nichts tun? LWF 40: 8-11.

Müller J, Engel H \& Blaschke M (2007) Assemblages of wood-inhabiting fungi related to silvicultural 
management intensity in beech forests in southern Germany. European Journal of Forest Research 126: 513-527.

Nilsson T (1973) Studies on wood degradation and cellulolytic activity of microfungi. Studia Forestalia Suecica 104.

Nordén B, Götmark F, Ryberg M, Paltto H \& Allmér J (2008) Partial cutting reduces species richness of fungi on woody debris in oak-rich forests. Canadian Journal of Forestry Research 38: 1807-1816.

Nordén B, Ryberg M, Götmark F \& Olausson B (2004) Relative importance of coarse and fine woody debris for the diversity of wood-inhabiting fungi in temperate broadleaf forests. Biological Conservation 117: 1-10.

Osono T \& Takeda H (2001) Organic chemical and nutrient dynamics in decomposing beech leaf litter in relation to fungal ingrowth and succession during 3-year decomposition processes in a cool temperate deciduous forest in Japan. Ecological Research 16: 649-670. doi:10.1046/j.14401703.2001.00426.x.

Ottosson E, Nordén J, Dahlberga A, Edmand M, Jönsson M, Larsson KH, Olssong J, Penttilä R, Stenlid J \& Ovaskainen O (2014) Species associations during the succession of wood-inhabiting fungal communities. Fungal Ecology 11: 17-28. doi:org/doi:10.1016/j.funeco.2014.03.003.

Ovaskainen O, Nokso-Koivisto J, Hottola J, Rajala $\mathrm{T}$, Pennanen T, Ali-Kovero $\mathrm{H}$, Miettinen $\mathrm{O}$, Oinonen P, Auvinen P, Paulin L, Larsson K-H \& Mäkipää R (2010) Identifying wood-inhabiting fungi with 454 sequencing - what is the probability that BLAST gives the correct species? Fungal Ecology 3: 274-283. doi:org/10.1016/j.funeco.2010.01.001.

Ovaskainen O, Schigel D, Ali-Kovero H, Auvinen P, Paulin L, Norden B \& Norden J (2013) Combining high-throughput sequencing with fruit body surveys reveals contrasting life-history strategies in fungi. International Society for Microbial Ecology Journal 7: 1696-1709. doi:10.1038/ismej.2013.61.

Panayotov K (2016) Influence of deadwood on soil carbon, nitrogen, bulk density and $\mathrm{pH}$ in a deciduous non-intervention forest reserve. Master's Thesis, University of Copenhagen, Copenhagen, pp. 1-51.

Pasinelli K \& Suter W (2000) Lebensraum Totholz. Merkblatt für die Praxis. WSL, Birmensdorf.

Penttilä R, Siitonen J \& Kuusinen M (2004) Polypore diversity in managed and old-growth boreal Picea abies forests in southern Finland. Biological Conservation 117: 271-283. doi:org/10.1016/j.biocon.2003.12.007.

Philpott TJ, Prescott CE, Chapman WK \& Grayston SJ (2014) Nitrogen translocation and accumula- tion by a cord-forming fungus (Hypholoma fasciculare) into simulated woody debris. Forest Ecology and Management 315: 121-128. doi:10.1016/j. foreco.2013.12.

Pichler V, Homolák M, Skierucha W, Pichlerová M, Ramírez D, Gregor J \& Jaloviar P (2012) Variability of moisture in coarse woody debris from several ecologically important tree species of the temperate zone of Europe. Ecohydrology 5: 424-434.

Pouska V, Svoboda M \& Lepšová A (2010) The diversity of wood-decaying fungi in relation to changing site conditions in an old-growth mountain spruce forest, Central Europe. European Journal of Forest Research 129: 219-231.

Prillinger H, Lopandic K, Sugita T \& Wuczkowski M (2007) Asterotremella gen. nov. albida, an anamorphic tremelloid yeast isolated from the agarics Asterophora lycoperdoides and Asterophora parasitica. The Journal of General and Applied Microbiology 53: 167-175.

Radu S (2007) The ecological role of deadwood in natural forests: Nature conservation: concept and practice (ed. by D Gafta, \& J Akeroyd) Springer, Berlin, pp. 137-141.

Rajala T, Peltoniemi M, Hantula J, Mäkipää R \& Pennanen T (2011) RNA reveals a succession of active fungi during the decay of Norway spruce logs. Fungal Ecology 4: 437-448. doi:org/10.1016/j.funeco.2011.05.005.

Rajala T, Peltoniemi M, Pennanen T \& Mäkipää R (2012) Fungal community dynamics in relation to substrate quality of decaying Norway spruce ( $P i$ cea abies [L.] Karst.) logs in boreal forests. FEMS Microbiology Ecology 81: 494-505. doi:10.1111/ j.1574-6941.2012.01376.x.

Rajala T, Peltoniemi M, Pennanen T \& Mäkipää R (2010) Relationship between wood-inhabiting fungi determined by molecular analysis (denaturing gradient gel electrophoresis) and quality of decaying logs. Canadian Journal of Forest Research 40: 2384-2397.

Rajala T, Tuomivirta T, Pennanen T \& Mäkipää R (2015) Habitat models of wood inhabiting fungi along a decay gradient of Norway spruce logs. Fungal Ecology 18: 48-55. doi:org/10.1016/j.funeco.2015.08.007.

Réblová M \& Stepánek V (2009) New fungal genera, Tectonidula gen. nov. for Calosphaeria-like fungi with holoblastic-denticulate conidiogenesis and Natantiella gen. nov. for three species segregated from Ceratostomella. Mycological Research 113: 991-1002. doi:10.1016/j.mycres.2009.06.003.

Renvall P (1995) Community structure and dynamics of wood-rotting Basidiomycetes on decomposing conifer trunks in northern Finland. Karstenia 35: 1-51. 
Ruetze M \& Parameswaran N (1984) Observations on the colonization of oak wilt mats (Ceratocystis fagacearum) by Pesotum piceae. Forest Pathology 14: 326-333.

Schmidt O (2006) Wood and tree fungi: biology, damage, protection and use. Springer-Verlag, Berlin, Heidelberg.

Schuck A, Meyer P, Menke N, Lier M \& Lindner M (2004) Forest biodiversity indicator: dead wood - a proposed approach towards operationalising the MCPFE indicator. EFI-Proceedings 51: 49-77.

Schwarze FWMR, Engels J \& Mattheck C (2000) Fungal strategies of wood decay in trees. Springer-Verlag, Berlin, Heidelberg.

Siitonen J (2001) Forest management, coarse woody debris and saproxylic organisms: fennoscandian boreal forests as an example. Ecological Bulletins 49: 11-41.

Skilling DD \& Walla JA (1986) Rhizosphaera needle cast of spruce: Diseases of trees in the great plains (ed. by JW Riffle \& GW Peterson) General Technical Reports, pp. 124-125.

Speight MCD (1989) Saproxylic invertebrates and their conservation. Nature and Environment 42: $1-79$.

Spribille T, Thor G, Bunnell FL, Goward T \& Björk CR (2008) Lichens on dead wood: species-substrate relationships in the epiphytic lichen floras of the Pacific Northwest and Fennoscandia. Ecography 31: 741-750.

Stachura K, Bobiec A, Obidziñski A, Oklejewicz K \& Wolkowycki D (2007) Old trees and decaying wood in forest ecosystems of Poland. Old wwood. A toolkit for participants, Version 07, 05.08.2011. http://oldwood.dle.interia.pl/OW_07.pdffrom.

Stokland JN, Siitonen J \& Jonsson BG (2012) Biodiversity in dead wood. Cambridge University Press, Cambridge.

Stokland JN \& Meyke E (2008) The saproxylic database: an emerging overview of the biological diversity in dead wood. Revue d'Ecologie 10: 37-48.

Szewczyk J \& Szwagrzyk J (1996) Tree regeneration on rotten wood and on soil in old growth stand. Vegetatio 122: 37-46.

Tyler G (1991) Ecology of the genus Mycena in beech (Fagus sylvatica), oak (Quercus robur) and hornbeam (Carpinus betulus) forest of S Sweden. Nordic Journal of Botany 11: 111-121.

Ulbrichová I, Remeš J \& Zahradník D (2006) Development of the spruce natural regeneration on mountain sites in the Šumava Mts. Journal of Forest Science 52: 446-456.

van der Wal A, de Boer W, Smant W \& van Veen J (2007) Initial decay of woody fragments in soil is influenced by size, vertical position, nitrogen availability and soil origin. Plant and Soil 301: 189-201. doi:10.1007/s11104-007-9437-8. van der Wal A, Klein Gunnewiek PJA, De Hollander M \& De Boer W (2017) Fungal diversity and potential tree pathogens in decaying logs and stumps. Forest Ecology and Management 406: 266-273. doi:org/10.1016/j.foreco.2017.08.018.

van der Wal A, Klein Gunnewiek PJ, Cornelissen JHC, Crowther TW \& Boer W (2016) Patterns of natural fungal community assembly during initial decay of coniferous and broadleaf tree logs. Ecosphere 7: e01393.

van der Wal A, Ottosson E \& de Boer W (2015) Neglected role of fungal community composition in explaining variation in wood decay rates. Ecology 96: 124-133.

Vandekerkhove K, Keersmaeker De L, Menke N, Meyer P \& Verschelde P (2009) When nature takes over from man: Dead wood accumulation in previously managed oak and beech woodlands in North-western and Central Europe. Forest Ecology and Management 258: 425-435.

Vane CH, Drage TC \& Snape CE (2006) Bark decay by the white-rot fungus Lentinula edodes: polysaccharide loss, lignin resistance and the unmasking of suberin. International Biodeterioration \& Biodegradation 57: 14-23.

Vasiliauskas R, Vasiliauskas A, Stenlid J \& Matelis A (2004) Dead trees and protected Polypores in unmanaged north-temperate forest stands of Lithuania. Forest Ecology and Management 193: 355-370.

Vaz ABM, Fonseca PLC, Leite LR, Badotti F, Salim ACM, Araujo FMG, Cuadros-Orellana S, Duarte AA, Rosa C, Guilherme O \& Aristóteles Góes-Neto $G$ (2017) Using next-generation sequencing (NGS) to uncover diversity of wood-decaying fungi in neotropical atlantic forests. Phytotaxa 295: $1-21$.

Vorčák J, Merganič J \& Merganičová K (2005) Deadwood and spruce regeneration. Lesnická práce 5: 18-19.

Vorčák J, Merganič J \& Saniga M (2006) Structural diversity change and regeneration processes of the Norway spruce natural forest in Babia hora NNR in relation to altitude. Journal of Forest Science 52: 399-409.

Wang Q, Garrity GM, Tiedje JM \& Cole JR (2007) Naive Bayesian classifier for rapid assignment of rRNA sequences into the new bacterial taxonomy. Applied and Environmental Microbiology 73: 5261-5267.

Watkinson S, Bebber D, Darrah P, Fricker M, Tlalka M \& Boddy L (2006) The role or wood decay fungi in the carbon and nitrogen dynamics of the forest floor: Fungi in biogeochemical cycles (ed. GM Gadd) Cambridge University Press, Cambridge.

White TJ, Bruns T, Lee S \& Taylor J (1990) Amplification and direct sequencing of fungal ribosomal 
RNA genes for phylogenetics: PCR protocols: A guide to methods and applications (ed. by MA Innis, DH Gelfland, JJ Sninsky \& TJ White) Academic Press, New York, pp. 315-322.

Wojewoda W (2003) Krytyczna lista wielkoowocnikowych grzybów podstawkowych Polski. Szafer
Institute of Botany, Polish Academy of Sciences. Kraków, Poland.

Zielonka T (2006) Quantity and decay stages of coarse woody debris in old-growth subalpine spruce stands of the western Carpathians, Poland. Canadian Journal of Forest Research 36: 2614-2622. 